\title{
Propagation of a gravity current in a two-layer stratified environment
}

\author{
P. Samothrakis and A. J. Cotel \\ Department of Civil and Environmental Engineering, University of Michigan, Ann Arbor, Michigan, USA \\ Received 24 June 2005; revised 23 September 2005; accepted 14 November 2005; published 21 January 2006.
}

[1] Gravity currents are created by a continuous release of dense fluid along a $6^{\circ}$ inclined ramp. The surrounding fluid is composed of a two-layer stably stratified environment. A chemical (phenolphthalein) reaction technique and Planar Laser Induced Fluorescence (PLIF) are used to measure the entrainment rate of lower fluid into the gravity current. The change of volume in the head of the gravity current quantifies the entrainment and mixing. Their dependence on the Richardson number, based on the gravity current head size and velocity and the initial density difference between the current and bottom layer fluids, is determined using chemically reacting PLIF. To further understand the entrainment process and to quantify the effect of the interface on the internal structure of the gravity current, Particle Image Velocimetry (PIV) measurements are performed. Both instantaneous velocity and vorticity fields are quantified. In addition, vertical profiles of velocity and vorticity are computed at two different locations, upstream and downstream of the impingement region. The vortical structure of the gravity current is clearly affected by the presence of the stratified interface. The level of restructuring depends on the Richardson number, based on the density difference across the interface. At low Ri, vorticity increases following the impingement, whereas at higher Ri, a decrease in vorticity is observed as well as a significant change in the head structure of the gravity current.

Citation: Samothrakis, P., and A. J. Cotel (2006), Propagation of a gravity current in a two-layer stratified environment, J. Geophys. Res., 111, C01012, doi:10.1029/2005JC003125.

\section{Introduction}

[2] Gravity currents are ubiquitous in the geophysical environment. A typical occurrence of such flows in the ocean is in the form of turbidity currents. They are masses of water and sediment that flow down the continental slope. They are responsible for the transfer of sediment from the continents to the oceans and the filling of large sedimentary basins at the bottom of the ocean. In many cases they are responsible for the breaking of submarine cables [Simpson, 1997]. A similar phenomenon can appear at the edges of lakes or that of continental shelves during winter. The temperature of the shallow waters at these locations falls more rapidly than that of the deeper waters. This temperature variation can form a cold and dense layer of fluid that flows (cascades) down the sides of lakes, creating a gravity current [Fer et al., 2002]. This winter cascading is sometimes strong enough to erode and transport suspended sediment from the shallow nearshore regions to deeper waters.

[3] Gravity currents also occur in the atmosphere, in the form of katabatic winds [Thompson, 1984]. The following problem describes such a case. Consider a large city in a valley where atmospheric conditions have created a strong

Copyright 2006 by the American Geophysical Union. 0148-0227/06/2005JC003125 inversion above the city. The pollution levels can become extremely high causing people to suffer from a variety of respiratory problems. If the katabatic winds are not strong enough to penetrate through the inversion and dilute the polluted air above the city, pollution levels will keep rising. Therefore, it is essential to quantify the physical processes taking place at the interface. Transport models with an accurate representation of entrainment need to be developed, to correctly predict pollution levels and design efficient countermeasures.

[4] Other examples of gravity currents that are encountered in nature include snow avalanches [Hopfinger and Tochon-Danguy, 1977] and pyroclastic flows [Valentine, 1987]. There are also examples of man-made gravity currents with industrial applications. Such an example is the instantaneous release of a dense gas in a less dense environment, after the failure of a containment tank [Baines, 2001]. Simpson [1997] has detailed descriptions of these and other examples of gravity currents and also provides an overview of the research performed on the subject.

[5] Having these examples in mind, the bottom surface over which oceanic and atmospheric currents move is not flat (for example the propagation of turbidity currents down the continental slope and of katabatic winds down a mountain slope). Also, the presence of a stratified environment in nature is a very common phenomenon (a thermocline in the ocean or an inversion in the atmosphere). Such a 
combination of conditions (sloping bottom and stratified interface) is encountered, for example, when rivers are discharging in the Mediterranean with its deep saline pools. In this paper, we focus on the specific case of a gravity current propagating on a slope and impinging on a stratified interface.

\section{Background}

[6] The research on gravity current dynamics and structure is extensive [Simpson, 1997]. Some of the early work on gravity currents was done by Benjamin [1968]. He proposed a theoretical approach based on inviscid fluid theory and provided solutions for depth and velocity of a gravity current. Previous experiments were performed in a uniform environment along the bottom of a tank [Simpson, 1982; Hallworth et al., 1993, 1996]. They performed measurements of the growth rate and hydrodynamic instabilities of gravity currents. Simpson [1982] described the gravity current as a succession of clefts and lobes, due to gravitational instability of the less dense fluid overrun by the nose of the current. Kelvin-Helmholtz instabilities are present in the region behind the head, creating intense mixing.

[7] In this paper entrainment is defined as engulfment of tongues of fluid [Roshko, 1976]. Entrainment is different from mixing, as fluid can be entrained but not mixed at the molecular level. This might happen for lower Reynolds numbers for example [Koochesfahani and Dimotakis, 1986] or in cases where fluid is entrained at some location of the flow and subsequently detrained before molecular mixing takes place. Entrainment measurements have been performed for 2-D and axisymmetric gravity currents along a flat surface in a uniform environment, i.e. no horizontal stratification, by Hallworth et al. [1996]. The gravity currents were created by the release of constant volume of dense fluid. The entrainment in the head of gravity current was found to only depend on the initial volume of the current and the distance from the release point. The entrainment was found to be independent of g' and therefore of the Richardson number. The entrainment coefficient was different between 2-D and 3-D currents, and varies with the surface of propagation, a smooth surface yielded a different entrainment constant than a rough surface [Hallworth et al., 1996].

[8] For gravity currents down a slope in a homogeneous environment, i.e. no density stratification, Turner [1973] found that

$$
E=R i_{o} \tan \vartheta
$$

where $\mathrm{E}$ is the entrainment; $R i_{o}$ is the overall Richardson number, defined by the velocity and height of the gravity current and the density difference between the gravity current and the ambient fluid, and $\vartheta$ is the slope angle. Friction on the wall can be neglected for slope angles $\vartheta$ (with respect to the horizontal) greater than a few degrees [Beghin et al., 1981]. A more general equation for entrainment is given by Turner [1973] when friction is taken into account:

$$
E=\frac{S_{2} R i \tan \vartheta-C_{D}}{1+\frac{1}{2} S_{1} R i}
$$

where $C_{D}$ is the drag coefficient and $S_{1}, S_{2}$ are two profile constants.

[9] The dynamics of a gravity current flowing downslope is changed dramatically from a horizontally propagating current [Hopfinger and Tochon-Danguy, 1977]. Ross et al. [2002] studied the effect of a sloping boundary on a threedimensional gravity current. They concluded that even relative gentle slopes $\left(5^{\circ}\right.$ to $\left.20^{\circ}\right)$ have a significant effect. Britter and Linden [1980] considered the flow of a gravity current from a continuous source down a slope in a uniform environment. The entrainment into the head increases with increasing slope. Also, they found that the velocity of the head $U_{f}$, non-dimensionalized by the cube root of the buoyancy flux $\left(g^{\prime} Q\right)^{1 / 3}$, is independent of the slope angle and equal to a constant as follows:

$$
\frac{U_{f}}{\left(g^{\prime} Q\right)^{1 / 3}}=1.5 \pm 0.2
$$

This is valid for the range of slopes $5^{\circ} \leq \vartheta \leq 90^{\circ}$. Also for $\vartheta \leq 5^{\circ}$ the head decelerates with distance from the source, because the buoyancy force is not strong enough to overcome the effect of friction [Beghin et al., 1981].

[10] Bonnecaze et al. [1995] and Bonnecaze and Lister [1999] studied the dynamics and deposition from particle driven currents on horizontal and sloped surface respectively. In the case of the horizontal surface, the flow was modeled by the single-layer axisymmetric shallow water equations, which succeeded in simulating adequately the mass, momentum and particle balances of the current. Hallworth et al. [1998] used a two-layer model incorporating the appropriate shallow-water equations to simulate the motion of the current and the deposition of particles. The results were found in good agreement with experimental data. Parker et al. [1987] performed experiments with turbidity currents over an erodible bed. By taking measurements of vertical profiles of velocity and sediment concentration, they developed approximate similarity relations.

[11] Monaghan et al. [1999] studied the case of gravity currents descending a ramp in a two-layer stratified tank, which matches our experimental set-up. Their interest was on the creation of internal waves along the stratified interface. Different ramp angles of $20^{\circ}, 45^{\circ}$ and $90^{\circ}$ degrees were used. The results of a numerical model, using the Lagrangian particle method, were compared to the experiments to explain the behavior of large amplitude waves. Sutherland et al. [2004] and Flynn and Sutherland [2004] used a two-layer stratified environment for their studies. In both cases, the gravity current was propagating along the interface. Sutherland et al. [2004] compared the prediction from an analytical solution for the speed and the vertical extent of the gravity current head with experimental results. They found an excellent agreement when the density of the gravity current is the average of the upper and lower-layer densities. Flynn and Sutherland [2004] studied the vertical flux of horizontal momentum due to the generation of internal waves along the interface and related it to properties of the fluid intrusion. As can be seen from this review of previous work, no measurements of entrainment have been per- 


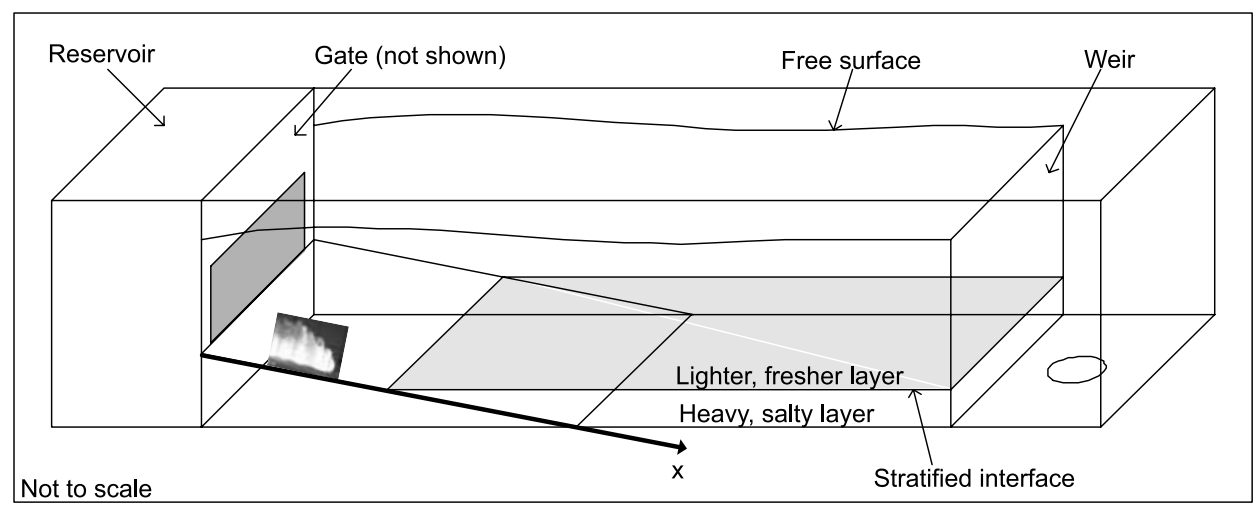

Figure 1. Schematic of the experimental apparatus.

formed in the case of a gravity current on a slope impinging on a stratified interface.

[12] Particle Image Velocimetry (PIV) measurements were performed by Alahyari and Longmire [1996] to study the structure of the head of an axisymmetric gravity current along a horizontal surface. They obtained vertical and horizontal velocity fields of the flow and observed a coherent pattern of large scale vortices. To study the structure of the head, Thomas et al. [2003] used a similar technique (Particle-Tracking Velocimetry), for the case of a 2-D gravity current. They acquired measurements of velocity, vorticity, strain stress at the head. Both experimental studies were performed in a uniform environment. No PIV measurements have been reported for a gravity current propagating in a 2-layer stratified environment.

[13] In this paper we investigate the flow resulting from the continuous release of a gravity current on a slope in a two-layer stratified environment. The objectives of this work are to quantify the effect of the stratified interface on the entrainment and mixing processes of a gravity current, and to investigate the effect of the interface on the structure of the gravity current head by providing detailed velocity and vorticity measurements.

\section{Experimental Setup}

[14] The experiments are performed in a $5 \mathrm{~m}$ long horizontal flume. The cross section of the flume is $30 \mathrm{~cm}$ wide by $50 \mathrm{~cm}$ high (Figure 1). The sloping bottom where the current is propagating is made of a thin Plexiglas sheet caulked to the sides of the flume. The slope is set at an angle of $6^{\circ}$ and has a length of approximately $2 \mathrm{~m}$. The $\mathrm{x}$-axis is considered to be along the sloping bottom, with $\mathrm{x}=0$ being at the origin which is represented by the location of the gate. The location of the stratified interface is at $\mathrm{x}=1.0 \mathrm{~m}$ and the end of the slope (where it becomes horizontal) is at $\mathrm{x}=2.0 \mathrm{~m}$. Saline solutions are used to achieve the density differences necessary to reach a given range of Richardson numbers. The density of the gravity current fluid varied between 1012 to $1100 \mathrm{~kg} / \mathrm{m}^{3}$, while the density of the bottom layer varied between 1005 to $1020 \mathrm{~kg} / \mathrm{m}^{3}$. The density of the gravity current fluid is higher than that of the lower layer to ensure a penetrating gravity current and not a propagating intrusion along the interface.

\subsection{Procedure}

[15] The flume is filled with a lighter, fresher layer first to a height of approximately $35 \mathrm{~cm}$. Then, the lower denser layer is set in place using a diffuser, connected to a supply tank of heavy, salty water. The diffuser is made of a thin Plexiglas plate glued to a short PVC pipe into which the salty water is supplied. This procedure prevents mixing between the two layers and creates a thin stable stratified interface with a thickness of approximately $0.5 \mathrm{~cm}$. The thickness of the interface was measured with a salinity probe. A typical vertical density profile is presented in Figure 2, where the difference between the density measured by the salinity probe and fresh water is plotted against the depth in the tank.

[16] The total height of fluid in the flume is $46 \mathrm{~cm}$ before the release of the gravity current fluid. An overflow weir was placed at the downstream end of the flume, in order to maintain a constant depth in all experiments. A lock release mechanism is used to generate two-dimensional gravity currents in the laboratory. The reservoir containing the gravity current fluid is connected to a pressure tank. By controlling the pressure in the tank, we have a constant head in the gravity current reservoir. During the filling process of the gravity current reservoir, a gate is locked in place to

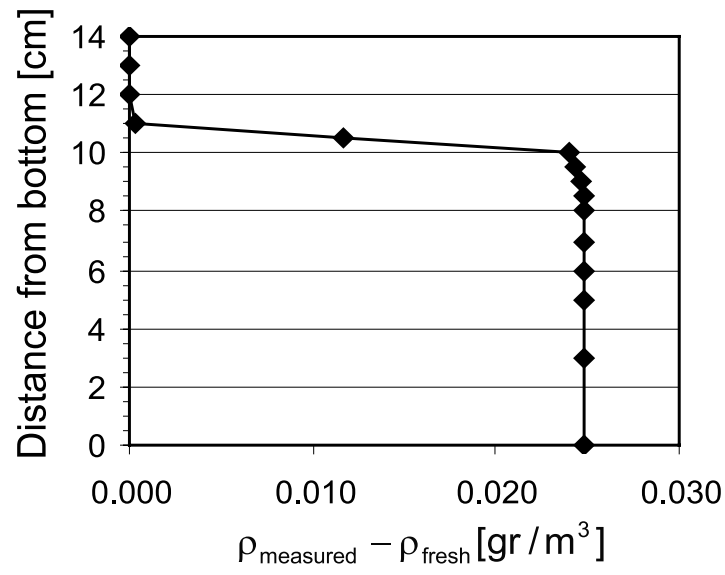

Figure 2. Typical density profile before the initiation of an experiment. 
separate the tank containing the two layers of salt water from the reservoir; therefore no initial mixing takes place between the gravity current and the stratified environment. Once the reservoir is full with the gravity current fluid, the gate is lifted to start an experiment. There is minimal initial momentum introduced in the flow, buoyancy rapidly takes over and the gravity current is purely gravity-driven at the moment of impingement.

\subsection{Parameters}

[17] Before proceeding to the description of the experiments, the parameters that govern the propagation of a gravity current on a slope at an angle $\vartheta$ have to be defined.

[18] For the series of experiments performed in this study the Reynolds number is in the range of 5000 to 14,000 and is defined as

$$
R e=\frac{U_{f} d}{\nu}
$$

where $U_{f}$ and $d$ are the characteristic velocity and height of the head of the gravity current before the impingement and $v$ is the kinematic viscosity of water.

[19] Following the same approach the Richardson number is based on the characteristics of the head before the impingement:

$$
R i=g \frac{\Delta \rho}{\rho} \frac{d}{U_{f}^{2}} \cos \vartheta
$$

where $g$ is the acceleration of gravity and $\Delta \rho$ is a density difference. Since there are three fluids with different densities among each other, the Richardson number can be defined in three different ways depending on the definition of $\Delta \rho$. The first one, $R i_{(C-T)}$, is defined by the density difference between the current and top layer fluids. This definition of $R i$ number determines the velocity and height of the head of the gravity current before the impingement, as the current initially encounters the top layer. If the current were to propagate in a uniform environment only this definition of Richardson number would be relevant. This $R i$ reflects the balance between the initial buoyancy and the characteristics of the gravity current. In that case the size and velocity of the current's head are entirely defined by the density difference between the current and the environment (here the top layer). The second one, $R i_{(B-C)}$, is defined by the density difference between the current and bottom layer fluids. After the impingement, the gravity current encounters the bottom layer fluid and their density difference is taken into account through $R i_{(B-C)}$. This definition of $R i$ number, as we will see in section 4.1, governs the rate by which bottom-layer fluid is entrained into the gravity current. Finally the third one, $R i_{(B-T)}$, is defined by the density difference between the bottom and top layer fluids. This definition of $R i$ number represents the relative strength of the interface with respect to the energy of the gravity current acquired before the impingement and the easiness by which the gravity current penetrates the interface.

[20] The range of the $R i$ number is highly affected by the velocity of the gravity current (which is squared in the definition). When a high density difference between the gravity current fluid and the ambient layers is used, the velocity is high and we get small $R i$ numbers. With a small density difference between the gravity current fluid and the ambient layers, the velocity is small and the resulting $R i$ number is high. As our interest is on penetrating gravity currents, there is a limit in the density difference that can be used and therefore the upper limit of the range of $R i$ is dictated by the physical constraints of the experimental set-up.

\subsection{Experimental Techniques \\ 3.3.1. Chemical Reaction Technique}

[21] A series of experiments was performed using a chemical reaction technique, with two main objectives: First, to visually observe the mechanisms that are responsible for the mixing and entrainment at the head of the current during the impingement and second, to quantify and relate these processes to the flow conditions. This technique takes advantage of the properties of phenolphthalein $\left(\mathrm{C}_{20} \mathrm{H}_{14} \mathrm{O}_{2}\right)$.

[22] Phenolphthalein is a $\mathrm{pH}$ indicator, which gives a red product strongly visible even for dilute concentrations at high $\mathrm{pH}$. The reactants are unobtrusive and the turbulence remains unaffected by the chemical reaction, there is no effect from surface tension or heat release. The transition interval for phenolphthalein is from about $\mathrm{pH}=8$ (clear) to $\mathrm{pH}=10.5$ (red). Under certain conditions the reaction is irreversible and used to quantify molecular level mixing [Breidenthal, 1981]. In this series of experiments, phenolphthalein and sulfuric acid $\left(\mathrm{H}_{2} \mathrm{SO}_{4}\right)$ are added to the gravity current fluid making the $\mathrm{pH}$ of the fluid below 4 . The $\mathrm{pH}$ of the bottom layer is typically fixed at 11 by the addition of a small amount of sodium hydroxide $(\mathrm{NaOH})$. Two cameras (one analog and one digital) recorded the motion of the gravity current along the slope. The first one provides information about the velocity and the size of the head and it is placed upstream of the interface. The second located at the interface, provides information about the mixing between the gravity current fluid and the bottom layer. A typical picture of the gravity current head before and after the impingement is shown in Figure 3 where the characteristic red product due to the presence of phenolphthalein can be seen.

\subsubsection{Planar Laser-Induced Fluorescence (PLIF) Technique}

[23] In order to further investigate the mixing and entrainment processes at the region of impingement, the Planar Laser-Induced Fluorescence (PLIF) technique was performed. Because the flow is three dimensional, the phenolphthalein does not provide a detailed visualization of the flow structure. The use of PLIF enabled us to visualize two-dimensional slices within the flow and provide information about the internal structure of the gravity current. The technique makes use of dyes whose fluorescence can be induced by a specific chemical environment. This chemical reaction is similar to the phenolphthalein technique described above, where acid and base are added to the fluids, with the fluorescent dye mixed in the acid solution. The dye fluoresces abruptly, when it is in a chemical environment with a $\mathrm{pH}$ above 4 and is excited 


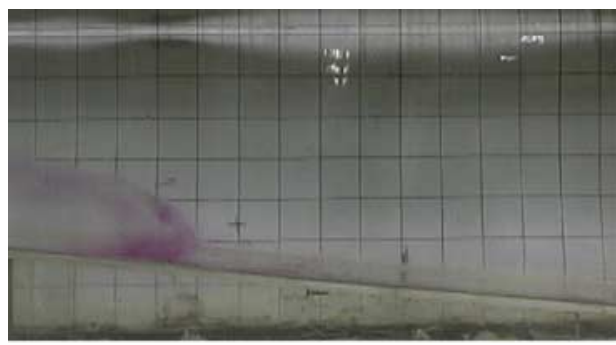

(a)

Figure 3. Gravity current before (a) and afte technique.

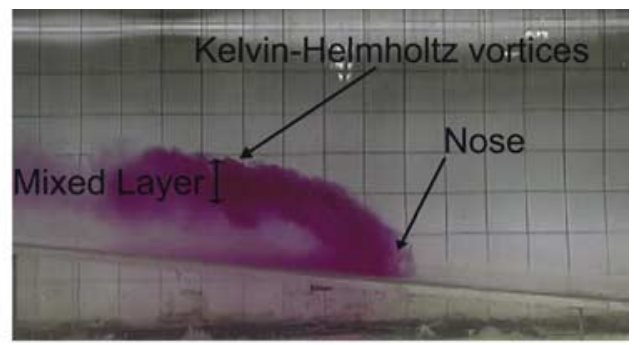

(b)

(b) the impingement with the chemical reaction

by an ion-argon laser with a wavelength between 488 and $514 \mathrm{~nm}$

[24] Disodium fluorescein, whose fluorescence is $\mathrm{pH}$ sensitive, was used in these experiments. Sulfuric acid $\left(\mathrm{H}_{2} \mathrm{SO}_{4}\right)$ and disodium fluorescein were added to the gravity current fluid and the $\mathrm{pH}$ of the solution was lowered to around 3. When sodium hydroxide $(\mathrm{NaOH})$ is added to the bottom layer, mixing between the different fluids is visualized with an ion-argon laser. The beam of the ion-argon laser is transformed into a thin sheet through a pair of cylindrical lens with focal length $\mathrm{f}=-12.7 \mathrm{~mm}$. The sheet is directed towards the tank using mirrors.

\subsubsection{Shadowgraph Experiments}

[25] Typical shadowgraph experiments were performed with and without the stratified interface, to investigate the effect of the stratified interface on the structure of the head. The light from a slide projector was diverted with a mirror towards the tank and projected on transparent paper. Pictures were taken at two different locations: at the point of impingement (where $\mathrm{x}=1.0 \mathrm{~m}$ ) and further downstream, at the end of the slope (where $\mathrm{x}=1.75 \mathrm{~m}$ ). The images were recorded on a digital camera.

\subsubsection{Particle Image Velocimetry Experiments}

[26] Particle Image Velocimetry (PIV) is an optical technique that provides high accuracy, non-intrusive planar flow measurements. The flow is quantitatively visualized through the use and motion of flow markers (particles). One issue with using optical techniques, like PLIF and PIV, in stratified flows is the difference in index of refraction between the different fluids. This problem can be alleviated by adding chemicals in the layers; for example, McDougall [1979] used an alcohol solution to match the indices of refraction in his experiments. Here the index of refraction was not an issue, because the density difference between the fluids was kept small.

[27] The gravity current fluid is seeded with titanium oxide $\left(\mathrm{Ti}_{2} \mathrm{O}\right)$ particles, with a size of $0.3 \mu \mathrm{m}$. These particles are neutrally buoyant in water. They have no time to settle or rise before an experiment as they are mixed into the gravity current fluid right before the initiation of an experiment. For the illumination of the particles a Nd:YAG laser (New Wave Research, Inc.) is used to form a sheet of green (532 nm) light. The laser light was directed from the bottom of the flume to the region of interest and a spherical and cylindrical lens were used to form a thin laser sheet $(0.75 \mathrm{~mm})$. The measurements are concentrated at the location of the impingement, spanning a distance of $25 \mathrm{~cm}$ upstream and downstream of the initial location of the stratified interface.

[28] The laser is pulsed twice at an interval of $5 \mathrm{msec}$, in order to ensure a particle displacement that is consistent with the spatial resolution and accuracy of the system. Simultaneously, a frame is obtained using a CCD camera, which exports the digital PIV images of the flow to the attached computer. The digitized images were analyzed using a PIV processing software (General Pixels Inc.). The software determined the velocity at points on an equally spaced grid covering the region of interest in the flow. The grid spacing used was 16 pixels, for a magnification factor of about 2 pixels $/ \mathrm{mm}$. Cross-correlation techniques [Adrian, 1986, 1991; Hinsch, 1993] applied to an interrogation window around the measurement point were used to determine the particle displacement field. For all the measurements, an interrogation window of size $32 \times 32$ pixels was used. Since the time interval between the two frames is known, the velocity of the particles can be deduced from their displacement. Based on the time between the laser pulses and the magnification factor, the error in the measured velocity is estimated to be $\pm 0.2 \mathrm{~cm} / \mathrm{sec}$. From the instantaneous velocity and vorticity fields, averages are computed over $0.2 \mathrm{sec}$.

\section{Results}

\subsection{Entrainment and Mixing}

[29] The details of the experimental conditions are given in Table 1 . The velocity of the gravity current, $U_{f}$ and the height $d$ of its head were measured for all the experiments, before the impingement, when the nose of the gravity current head is just touching the interface at $\mathrm{x}=1.0 \mathrm{~m}$. The following procedure was used: before a series of experiments, a ruler is placed at the location of the laser sheet and the magnification is calculated (in terms of pixels $/ \mathrm{cm}$ ). The number of pixels that correspond to a certain dimension on the picture (like the height $d$ of the head, or the location $x$ of the head) is obtained through image processing. Based on the size of the images $(776 \times$ 484 pixels), the uncertainty is estimated to be \pm 0.6 pixels or (with a typical magnification of $15 \mathrm{px} / \mathrm{cm}$ ) $\pm 0.4 \mathrm{~mm}$. In a similar way, for the experiments with phenolphthalein, the magnification was extracted by a $5 \times 5 \mathrm{~cm}$ grid placed on the back of the tank. The uncertainty in this case is 
Table 1. Velocity $U_{f}$ and Height $d$ of the Head Before the Impingement for the PLIF Experiments ${ }^{\mathrm{a}}$

\begin{tabular}{lccccc}
\hline Experiment & $U_{f}, \mathrm{~cm} / \mathrm{sec}$ & $\mathrm{d}, \mathrm{cm}$ & $\rho_{b}, \mathrm{~kg} / \mathrm{m}^{3}$ & $\rho_{c}, \mathrm{~kg} / \mathrm{m}^{3}$ & $\rho_{t}, \mathrm{~kg} / \mathrm{m}^{3}$ \\
\hline PLIF1 & 14.3 & 9.0 & 1,016 & 1,032 & 1,000 \\
PLIF2 & 15.0 & 8.5 & 1,020 & 1,040 & 1,000 \\
PLIF3 & 10.3 & 11.1 & 1,014 & 1,028 & 1,000 \\
PLIF4 & 10.1 & 10.1 & 1,010 & 1,022 & 1,000 \\
PLIF4H & 23.6 & 8.9 & 1,012 & 1,100 & 1,000 \\
PLIF5 & 7.8 & 8.9 & 1,010 & 1,013 & 1,000 \\
PLIF6 & 13.4 & 9.7 & 1,010 & 1,030 & 1,000
\end{tabular}

${ }^{a}$ Here $\rho_{b}, \rho_{t}, \rho_{c}$ are the densities of the bottom, the top layer, and the gravity current fluid, respectively.

estimated to be $\pm 1 \mathrm{~mm}$. The typical lobes and clefts structures [Simpson, 1997] are observed along the upper surface of the gravity current head. As a result fluctuations are seen in this area. When the flow is visualized with dye or shadowgraph, it is more difficult to define the height and location of the head. But for the measurements based on the PLIF images, where a slice of the flow is visualized, the height/location of the head is easily determined through image processing.

[30] The velocity of the gravity current, $U_{f}$, satisfies the ratio of equation (3) with the constant being equal to $1.6 \pm .3$ (Figure 4). This shows that the gravity current is well developed when it hits the interface and the effect of friction is negligible in our experiments.

[31] Hallworth et al. [1996] measured the entrainment for a two-dimensional, constant-volume gravity current, as the ratio of the volume of ambient fluid to the initial volume of the gravity current in the head. The volume of ambient fluid in the head was determined with the use of a similar chemical reaction technique. For the case of a constant-flux gravity current, Britter and Linden [1980], defined the entrainment as the rate of change of the head height.

[32] The entrainment is defined here as the change in the height of the mixed layer before, $h_{1}$, and after the impingement, $h_{2}$, (non-dimensionalized by the height of the mixed layer before the impingement, $h_{1}$ ). The mixed layer is the area in the flow, just behind the head of the current, where

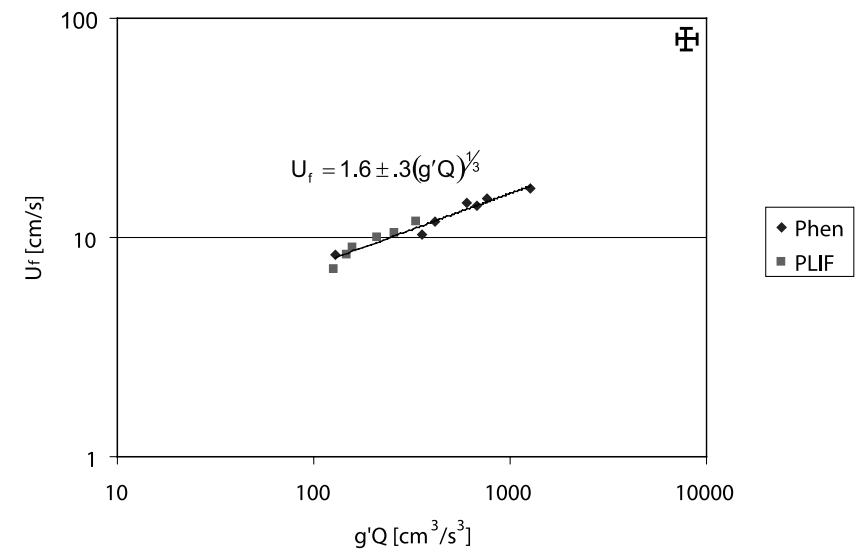

Figure 4. Velocity of the head $U_{f}$ as a function of buoyancy flux $\left(g^{\prime} Q\right)$.
Kelvin-Helmholtz eddies are present and entraining fluid (see red area in Figure $3 \mathrm{~b}$ ) and where the $\mathrm{pH}$ indicator (phenolphthalein) is turned on due to the occurrence of molecular level mixing. The height $h_{2}$ was measured around $80 \mathrm{~cm}$ downstream of the location of impingement, at $\mathrm{x}=$ $1.8 \mathrm{~m}$, with the procedure described earlier. In Figure 5 the entrainment is plotted versus the Richardson number and it is found to be proportional to $R i_{(B-C)}^{-1 \pm .1}$. Error estimates are provided in the upper right corner of the graph.

[33] The rate of growth of the head $d h / d x$ was measured from the Planar Laser Induced Fluorescence experiments as described earlier, with $h$ being the height of the head and $x$ the downstream distance along the slope. This ratio was measured at two different locations to ensure that the effect of the interface on the entrainment of the gravity current is considered. The first one is at a distance 10 to $30 \mathrm{~cm}$ after the interface, marked as 'After the impingement' in Figure 6 and the second one at a distance 60 to $80 \mathrm{~cm}$ after the interface marked as 'Downstream Location' in Figure 6. The rate of growth of the head at the two locations is plotted versus Richardson number and is found to be proportional to $R i_{(B-C)}^{-1 \pm .1}$ (Figure 6). As the gravity current is propagating through the bottom layer, the density difference of the two fluids controls the entrainment rate between them. Also, both the velocity and height of the gravity current before the impingement affect the entrainment rate. These parameters are implicitly included in $R i_{(B-C)}$. The relevant $R i$ is based on the difference between the current and the bottom layer. The entrainment we are interested in quantifying is the entrainment of lower or bottom layer fluid into the gravity current. Implicitly, the density of the top layer is important as it defines the gravity current velocity and size before impingement. Therefore, the relevant $R i$ combines the density of the gravity current, its size and velocity; and the density of the bottom layer. A different set of experiments was performed for the downstream location and thus the different number of points between the two locations in the graph. The experimental conditions for the downstream location were similar to those in Table 1.

[34] The shift in the measured values of $d h / d x$ between the two locations is very close to our estimated error. Part of the difference can be attributed to the fact that as the gravity current penetrates the interface, it encounters a denser fluid

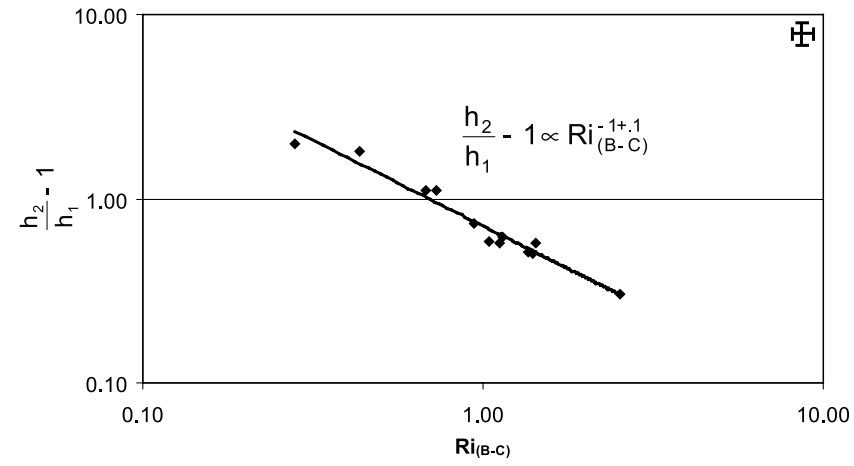

Figure 5. The change of height of the mixed layer, nondimensionalized by the height of the mixed layer before the impingement. 


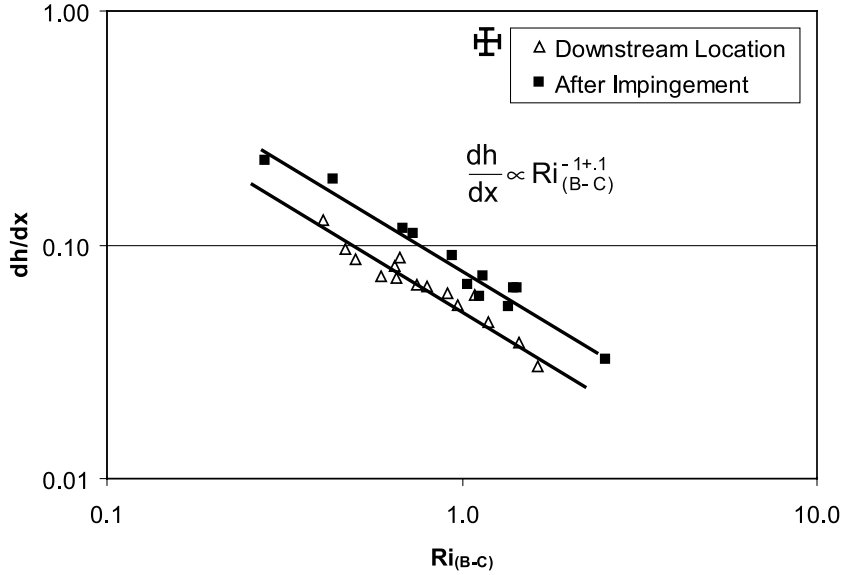

Figure 6. The rate of growth of the head versus Richardson number right after the impingement and in the downstream location.

than the one that it was propagating before. As seen in the next section, that causes a reduction in the gravity current velocity and a deepening in the head size. Finally, it should be noted that the range of variation of the $R i$ number is close to a decade. As noted in section 3.2, the range is limited due to constraints linked to the experimental apparatus.

[35] The gravity current entrains fluid from the top layer before the impingement. This case of a gravity current on a slope propagating on a uniform environment, corresponds to the study of Britter and Linden [1980] and Beghin et al. [1981] and it is not further investigated here. Monaghan et al. [1999] with a two-layer stratified environment, observed that due to entrainment before the impingement some gravity current fluid becomes buoyant relative to the bottom layer and flows along the interface. This is not observed in our case, as our slope is mild $\left(6^{\circ}\right)$ compared to the steep slopes $\left(20^{\circ}, 45^{\circ}\right.$ and $\left.90^{\circ}\right)$ used by Monaghan et al. [1999] and the entrainment is much higher for steeper slopes [Britter and Linden, 1980].

[36] Figure 7 shows a photographic sequence of the gravity current, visualized by the phenolphthalein technique. It can be seen from the images that there are two mechanisms responsible for mixing. The first one is the over-riding and engulfment of ambient fluid beneath the nose of the head. Figure 7a shows the location where we first observe the red product from the mixing of current and bottom fluids. There is no mixing at the front of the current. Similarly Johari [1992] for the case of a thermal observed that the volume of entrained fluid by the small-scale eddies on the front of the thermal is small compared to the entrainment by the large-scale eddies in the perimeter of the thermal. Furthermore, these large eddies are responsible for entraining surrounding fluid and bringing it to the center of the thermal where it is ultimately mixed. This is similar to the process of over-riding and engulfment in the case of the gravity current.

[37] The second mixing mechanism is due to the KelvinHelmholtz instabilities along the upper surface of the head [Britter and Simpson, 1978]. In Figure 7, we can clearly see the bright red color that appears on the upper surface of the head, an indication of intense mixing, which represent the location of the K-H vortices created by the difference in velocity between the environment and the gravity current.

[38] A similar photographic sequence of the gravity current taken with the Planar Laser Induced Fluorescence technique is shown in Figure 8. The same two mixing mechanisms (overriding of ambient fluid and the KelvinHelmholtz instabilities) that were observed from the phenolphthalein technique are also observed with the PLIF measurements. This confirms that the Kelvin-Helmholtz vortices, formed on the upper surface of the head, represent the dominant mixing mechanism. Similar observations have been reported in other turbulent flows by Brown and Roshko [1974], Dahm and Dimotakis [1987] and Papantoniou and List [1989]. In Figures 8c and 8d we can see more details of the flow, e.g. two large structures following one another.
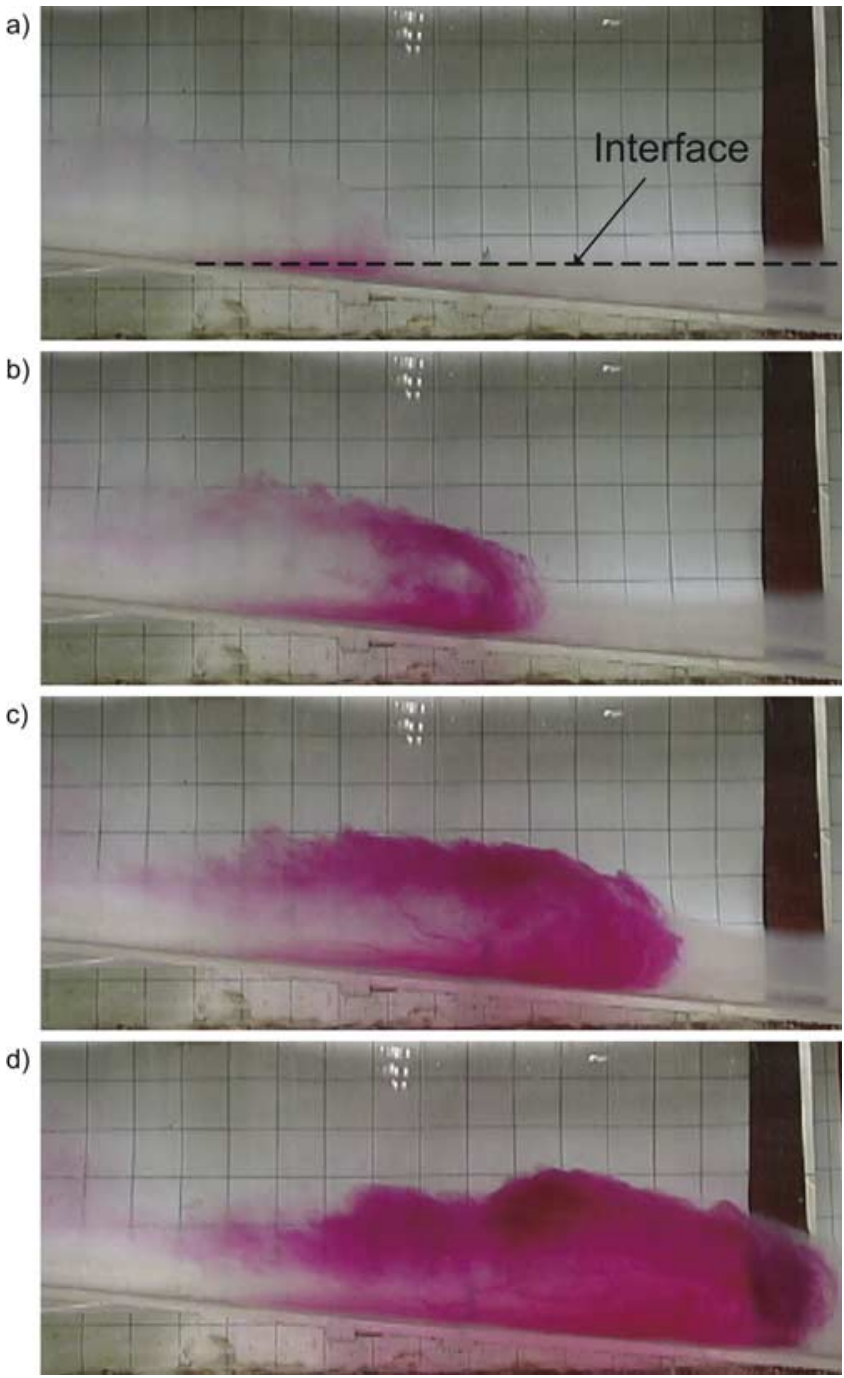

Figure 7. Sequence of images for the phenolphthalein technique $\left(\mathrm{Re}=9500\right.$ and $\left.R i_{(B-C)}=1.6\right)$. The images are $2 \mathrm{sec}$ apart. The square grid on the back is $5 \times 5 \mathrm{~cm}$. 
a)

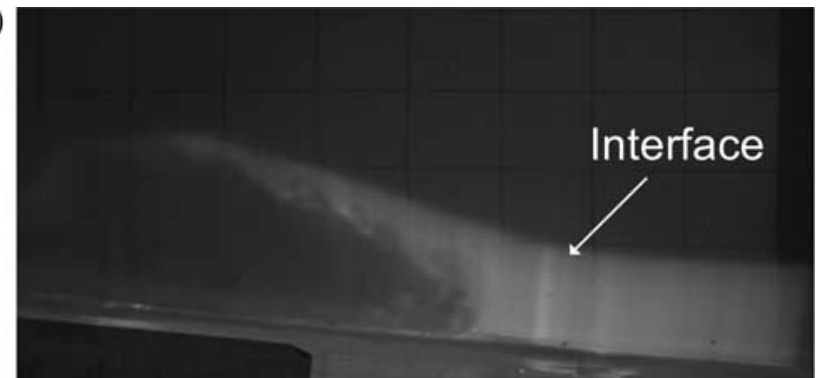

b)

c)


d)

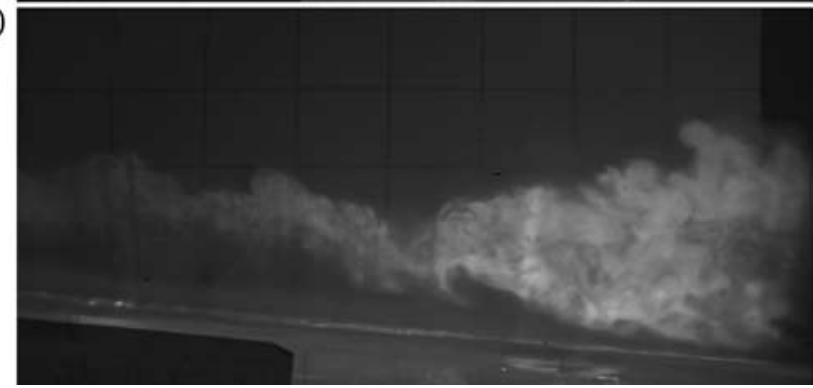

Figure 8. Sequence of images for the Planar Laser Induced Fluorescence technique $(\mathrm{Re}=10,500$ and $\left.R i_{(B-C)}=1.8\right)$.

\subsection{Structure of the Gravity Current Head}

[39] Figure 9 shows shadowgraph images of the front of the current at the two different locations: at the point of impingement (a) and further downstream (b), the image's edge being approximately $50 \mathrm{~cm}$ downstream from the point of impingement. From the images on the left column, we observe that the front of the current has a distinctive raised head structure [Britter and Linden, 1980]. But as the current propagates further downstream (Figure $9 b$ ), we observe that the size of the head is thickening and we cannot longer observe a distinctive head structure. This thickening of the head is not the result of higher entrainment of ambient fluid as demonstrated by the entrainment measurements. The fact that the gravity current is propagating in a denser environ- ment and encountering a stratified interface is causing the front to slow down and spread in the vertical direction.

[40] Figure 10 shows shadowgraph images of two separate experiments. The experimental conditions (density of gravity current fluid and buoyancy flux) are the same and the only difference is the presence of the stratified interface in the images of the right column (there is no stratified interface in the left column). Both sequences of images are recorded at the downstream location, close to the end of the slope. The left edge of the images is $50 \mathrm{~cm}$ downstream from the point of impingement. The sequence of images in the right column is the same as in Figure 9b, where we observed a change (thickening) in the size of the head. In the case where there is no stratified interface (Figure 10a), the thickening of the head is much less. The structure of the gravity current head is then similar to the stratified case before impingement (Figure 9a) and previously observed by Britter and Linden [1980].

\subsection{Velocity and Vorticity}

[41] A sample of the conditions for the Particle Image Velocimetry experiments are given in Table 2 . As before the velocity of the gravity current, $U_{f}$ and the height $d$ of its head were measured before the impingement for all the experiments with the procedure described in section 4.1.

[42] Figure 11 is a typical average vorticity field of the gravity current head. To get a better understanding of the structure, a shadowgraph of the head has been superimposed over the vorticity contours. The experimental conditions were similar for the PIV and shadowgraph images. The color bar on the bottom indicates the sign and magnitude of the vortices. The red color (positive vorticity) indicates a counterclockwise vortex, while the blue color (negative vorticity) indicates a clockwise rotating vortex.

[43] From Figure 11 we can make the following observations about the structure of the gravity current head. Negative vorticity (clockwise vortices) is created as the current is propagating over the inclination because of the no-slip boundary condition imposed there. A boundary layer is formed along the inclination. Eddies are created through the effect of viscous forces and can be readily observed in Figure 11. With the angle of the slope being greater than $5^{\circ}$, the effect of friction is negligible [Beghin et al., 1981]. On the other hand, the shear stress along the upper surface of the head and the presence of the KelvinHelmholtz instabilities can be observed as a region of positive vorticity. At the same time, as the dense gravity current fluid is propagating into the ambient fluid, baroclinic vorticity is created across the density interface. This kind of vorticity causes the fluid in the head to create a counterclockwise vortex (i.e. positive vorticity). It is impossible, at present, for us to differentiate between KelvinHelmholtz and baroclinic vorticity, since in this case, both resulting vortices are rotating in the same direction.

[44] From the instantaneous velocity and vorticity fields, averages are computed over $0.2 \mathrm{sec}$. These average fields are presented in two different locations, before the impingement (Figure 12a), when the gravity current head is just touching the interface and after the impingement, when the gravity current is just exiting the region of interest (Figure 12b). 

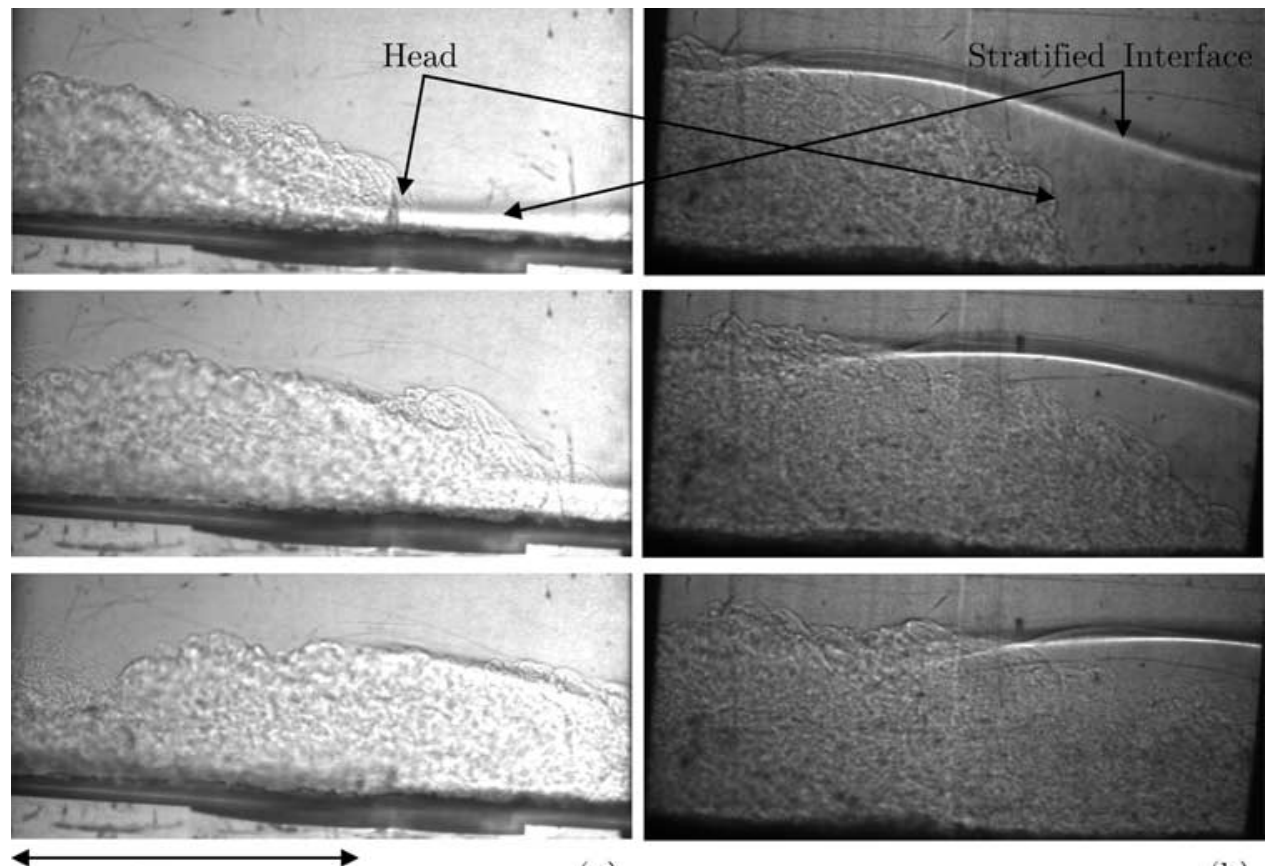

$25 \mathrm{~cm}$

(a)

(b)

Figure 9. Shadowgraph images of the head of the current at the impingement region (a) and further downstream, close to the end of the ramp (b). The images are $2 \mathrm{sec}$ apart.

[45] In the following Figures 13 and 14, average vorticity fields are presented for two different Richardson numbers. Here the Richardson number is based on the density difference between the bottom and top layer fluids $\left(R i_{(B-T)}\right)$. This definition of the Richardson number characterizes the strength of the stratified interface. The initial characteristics of the gravity current (velocity and height of the head) are determined by the density difference of the gravity current and top-layer fluids. The density difference across the interface controls the easiness by which the current is penetrating the interface and has an effect on the structure of the head. Figures $13 \mathrm{a}$ and $14 \mathrm{a}$ represent the vorticity field before the impingement (as seen in Figure 12a). Similarly Figures $13 \mathrm{~b}$ and $14 \mathrm{~b}$ represent the vorticity field after the impingement (as seen in Figure 12b). The location of the impingement with the stratified interface in all cases is at $\mathrm{x}=25 \mathrm{~cm}$.

[46] From the vorticity fields (PIV images), we can qualitatively observe that for the weakly stratified case (Figure 13), the strength of the vorticity is high to begin with (13a) and it increases after the impingement (13b). On the other hand, for the highly stratified case (Figure 14), the strength of the vorticity is much lower; especially for the counterclockwise vortices along the upper surface of the gravity current head, but there is a strong clockwise vortex along the slope (14a). After the impingement we observe that the strength of the vorticity decreases and the vortices tend to break up.

[47] To further quantify the effect of stratification on the structure of the gravity current head, vertical profiles of vorticity were taken at two different locations, approximately $10 \mathrm{~cm}$ before and $10 \mathrm{~cm}$ after the intersection of the interface with the slope. The 'before' was taken when the head is just touching the interface, as seen in Figure 15a and the 'after' when the head of the gravity current is approximately 10 centimeters before exiting the region of interest, as seen in Figure 15b. Also the location of the vertical profiles can be seen in Figures 13 and 14 (dotted lines) and Figure 16

[48] The change in vorticity was also quantified with the following approach: from the measured quantities $U_{f}$ and $d$ (Table 2) a time scale, $t_{L E}=\frac{d}{U_{f}}$, that corresponds to the rotation period of large-size eddies was evaluated. Then the maximum and minimum vorticity was extracted from the PIV data at the two locations mentioned above (Figure 15). The change in vorticity between the two locations was evaluated as follows:

$$
\begin{aligned}
& \Delta \omega^{+}=\omega_{\text {after }}^{+}-\omega_{\text {before }}^{+} \\
& \Delta \omega^{-}=\omega_{\text {after }}^{-}-\omega_{\text {before }}^{-}
\end{aligned}
$$

where $\omega_{\text {after }}^{+}$and $\omega_{\text {before }}^{+}$, represent the maximum (positive) vorticity after and before the impingement respectively and $\Delta \omega^{+}$their change. Similarly $\omega_{\text {after }}^{-}$and $\omega_{\text {before }}^{-}$, represent the minimum (negative) vorticity after and before the impingement respectively and $\Delta \omega^{-}$their change. Both $\Delta \omega^{+}$and $\Delta \omega^{-}$are then non-dimensionalized by the time scale $t_{L E}^{-1}$ and plotted versus the $R i_{(B-T)}$ in Figure 17. Error estimates are provided in the right upper corner of the graph.

[49] When $R i_{(B-T)}<1$, both $\Delta \omega^{+}$and $\Delta \omega^{-}$are positive, representing an increase of both positive and negative vorticity and this increase is becoming larger as $R i_{(B-T)}$ is decreasing (i.e. the stratification is weaker). The vorticity 

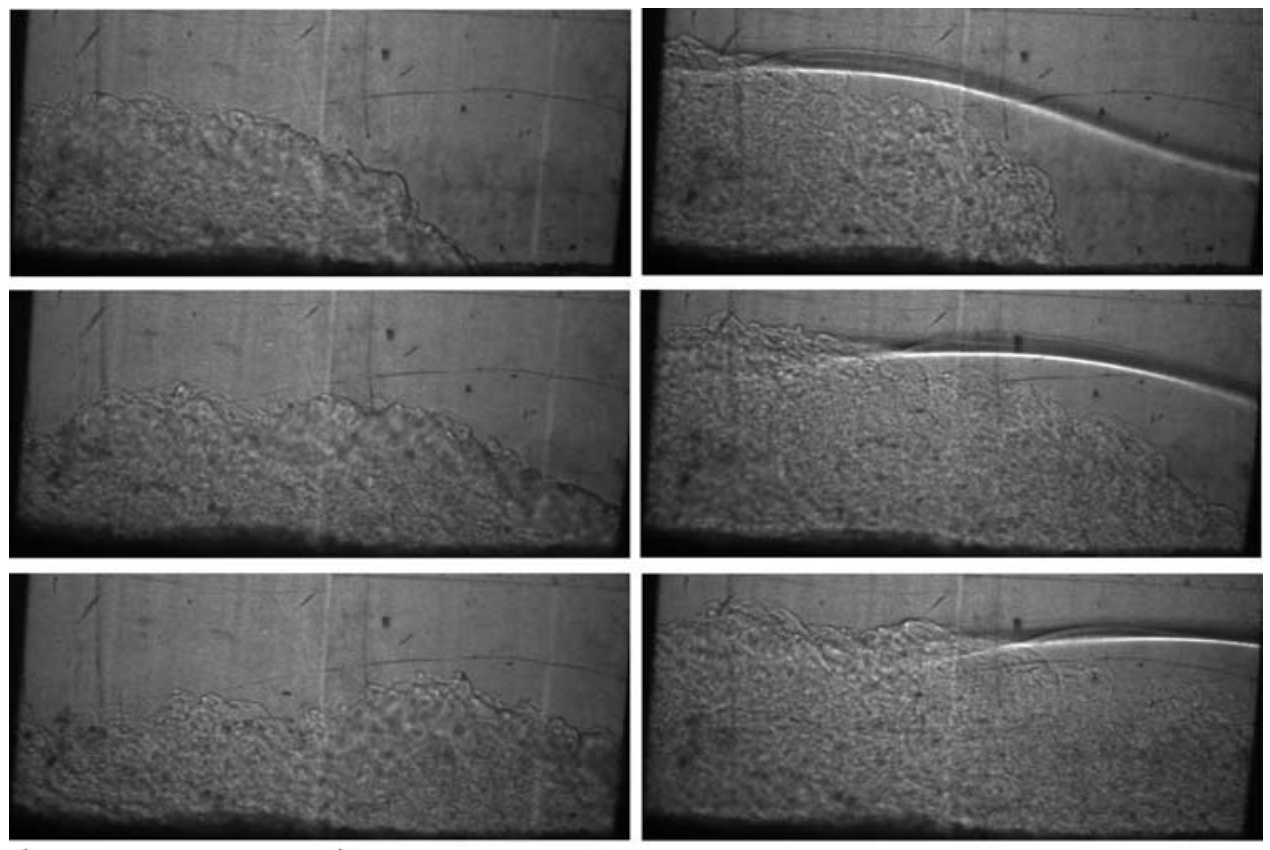

$25 \mathrm{~cm}$

(a)

(b)

Figure 10. Shadowgraph images of the head of the current at the downstream location with no stratification (a) and with the presence of the stratified interface (b). The images are 2 sec apart.

remains constant when $R i_{(B-T)}$ is around $1, \Delta \omega^{+}=0$ and $\Delta \omega^{-}=0$ for $R i_{(B-T)}=1$ as seen in Figure 17. When $R i_{(B-T)}$ $>1$, both $\Delta \omega^{+}$and $\Delta \omega^{-}$are negative, representing a reduction of both positive and negative vorticity. Moreover, this reduction is becoming larger with increasing $R i_{(B-T)}$, as the strength of the eddies is restrained by the presence of the strong stratified interface. The dependence between $\frac{\Delta \omega^{+}}{t_{L E}^{-1}}, \frac{\Delta \omega^{-}}{t_{L E}^{-1}}$ and $R i_{(B-T)}$ is found to be linear as seen in Figure 17:

$$
\begin{aligned}
& \frac{\Delta \omega^{+}}{t_{L E}^{-1}}=-1.02 \cdot R i_{(B-T)}+1.10 \\
& \frac{\Delta \omega^{-}}{t_{L E}^{-1}}=-1.22 R i_{(B-T)}+1.57
\end{aligned}
$$

[50] From the same experimental conditions, average velocity fields are evaluated and in Figure 18 the weakly stratified case is presented. The highly stratified case can be found in Samothrakis [2005]. The locations of 'before' and 'after' are the same as defined in Figure 12. The location of the impingement with the stratified interface in all cases is at $\mathrm{x}=$ $25 \mathrm{~cm}$. Moreover, vertical profiles of velocity (Figure 19) were taken at two different locations, $10 \mathrm{~cm}$ before and $10 \mathrm{~cm}$ after the stratified interface. The location of the vertical profiles can be seen in Figure 18 (dotted lines).

[51] From the velocity fields, we can observe that for the weakly stratified case (Figure 18), the velocity is high to begin with (18a) and it increases slightly after the impingement (18b). On the other hand, for the highly stratified case, after the impingement we observe that the velocity decreases due to the presence of the strong stratified interface [Samothrakis, 2005].

[52] These observations can be seen more clearly in the velocity profiles. For the weakly stratified case (Figures 19a and 19c, before and after the impingement, respectively) the velocity remains high, whereas in the highly stratified case (Figures 19b and 19d, before and after the impingement, respectively) we observe a significant decrease.

[53] The change in velocity was quantified with a similar approach to the vorticity: the maximum velocity was extracted from the PIV data at the two locations of the vertical profiles (Figure 15). The change in velocity between the two locations was evaluated as follows:

$$
\Delta u=u_{\text {after }}-u_{\text {before }}
$$

Table 2. Velocity $U_{f}$ and Height $d$ of the Head Before the Impingement for the PIV Experiments ${ }^{\mathrm{a}}$

\begin{tabular}{cccccc}
\hline Experiment & $U_{f}, \mathrm{~cm} / \mathrm{sec}$ & $\mathrm{d}, \mathrm{cm}$ & $\rho_{b}, \mathrm{~kg} / \mathrm{m}^{3}$ & $\rho_{c}, \mathrm{~kg} / \mathrm{m}^{3}$ & $\rho_{t}, \mathrm{~kg} / \mathrm{m}^{3}$ \\
\hline PIV1 & 10.8 & 7.7 & 1,007 & 1,025 & 1,000 \\
PIV2 & 9.4 & 8.1 & 1,008 & 1,014 & 1,000 \\
PIV3 & 7.7 & 10.0 & 1,006 & 1,015 & 1,000 \\
PIV4 & 7.9 & 8.2 & 1,011 & 1,019 & 1,000 \\
PIV5 & 6.7 & 8.4 & 1,010 & 1,016 & 1,000 \\
PIV6 & 6.0 & 8.9 & 1,008 & 1,010 & 1,000 \\
PIV7 & 5.8 & 11.4 & 1,009 & 1,012 & 1,000 \\
\hline
\end{tabular}

${ }^{a}$ Here $\rho_{b}, \rho_{t}, \rho_{c}$ are the densities of the bottom, the top layer, and the gravity current fluid, respectively. 

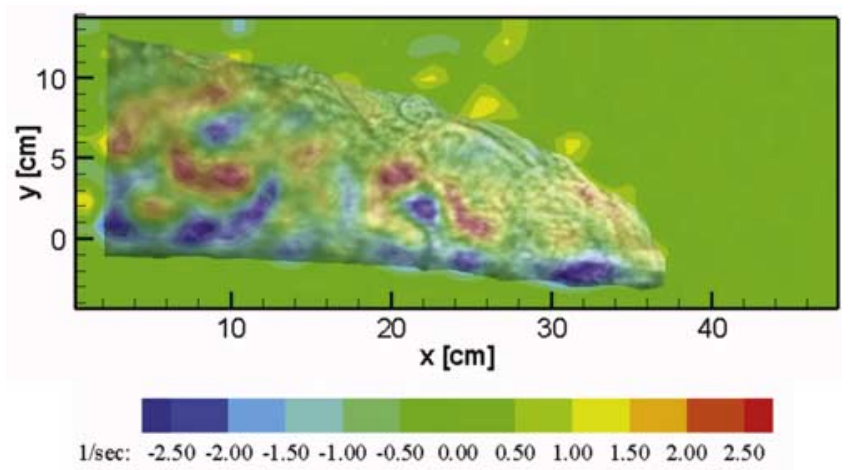

Figure 11. Typical vorticity field of the head. A shadowgraph of the head has been superimposed $(\mathrm{Re}=$ 7500 and $\mathrm{Ri}=1.3)$.

where $u_{\text {after }}$ and $u_{\text {before }}$, represent the maximum velocity after and before the impingement respectively and $\Delta u$ their change. $\Delta u$ is then non-dimensionalized by the velocity of the gravity current $U_{f}$ (Table 2 ) and plotted versus $R i_{(B-T)}$ in Figure 20. Error estimates are provided in the right upper corner of the graph.

[54] When $R i_{(B-T)}<1, \Delta u$ is positive, representing an increase of velocity and this increase is becoming larger as $R i_{(B-T)}$ is decreasing (i.e. the stratification is weaker). The velocity remains almost constant for the case that $R i_{(B-T)}$ is around 1 . When $R i_{(B-T)}>1, \Delta u$ is negative, representing a reduction in velocity. Moreover, this reduction is becoming larger with increasing $R i_{(B-T)}$, as it is more difficult for the gravity current to penetrate the interface. The dependence between $\frac{\Delta u}{U_{f}}$ and $R i_{(B-T)}$ is found to be linear as seen in Figure 20:

$$
\frac{\Delta u}{U_{f}}=-0.40 \cdot R i_{(B-T)}+0.42
$$

[55] Based on equation (3), the head of the gravity current has a constant velocity when flowing down a slope in a uniform environment [Britter and Linden, 1980]. Therefore, it is not immediately clear, why the velocity and vorticity are increasing for weakly stratified cases (when $R i_{(B-T)}<1$ ). In this range of $R i$, the gravity current velocity is high to start with because of a large density difference between the initial gravity current fluid and the top layer fluid. In all cases the gravity current is propagating into a denser fluid after the impingement and the velocity should decrease. In order to explain this, PIV experiments are performed in a uniform environment (i.e. with no stratification).

[56] The average vorticity and velocity fields of these experiments, similar to Figures 13,14 , and 18, can be found in Samothrakis [2005]. The vertical profiles of velocity for two unstratified cases are presented in Figure 21. In Figures $21 \mathrm{a}$ and $21 \mathrm{c}$, the gravity current fluid has a density of $\rho_{c}=1012 \mathrm{~kg} / \mathrm{m}^{3}$ while in Figures $21 \mathrm{~b}$ and $21 \mathrm{~d}$, the gravity current fluid has a density of $\rho_{c}=1022 \mathrm{~kg} / \mathrm{m}^{3}$. For both cases, the top layer is pure fresh water with $\rho=1000 \mathrm{~kg} / \mathrm{m}^{3}$.

[57] In the case of the low density gravity current (Figures 21a and 21c), there is no change in velocity. In both graphs the maximum velocity is around $5 \mathrm{~cm} / \mathrm{sec}$. The gravitational force down the slope is balanced by the frictional and entrainment drag, equation (3) is satisfied. On the other hand, in the case of the high density gravity current (Figures 21b and 21d), there is an increase in the velocity. In Figure $21 \mathrm{~b}$ the maximum velocity is around $6 \mathrm{~cm} / \mathrm{sec}$ and in Figure 21d around $6.7 \mathrm{~cm} / \mathrm{sec}$. In both graphs, equation (3) is satisfied within its margin of error.

[58] If a two-layer stratified environment was used in these experiments, Figures $21 \mathrm{a}$ and $21 \mathrm{c}$ would correspond to the highly stratified case as the velocity of the gravity current before the impingement is low. Similarly, Figures $21 \mathrm{~b}$ and $21 \mathrm{~d}$ would correspond to the weakly stratified case, as the velocity of the gravity current before the impingement is high.

[59] This can explain the increase in the velocity and vorticity observed in the stratified case, when $R i_{(B-T)}<1$. This $R i$ number corresponds to the case of high density difference between the gravity current fluid and the ambient environment. The velocity of the gravity current is high before the impingement and the density difference across the interface is small. The current easily penetrates the interface and its velocity slightly increases within the range of equation (3), until an equilibrium between the buoyancy force and drag due to entrainment and friction is reached. The increase in velocity and vorticity reflect adjustment of the flow to these conditions before equilibrium is reached.

\section{Discussion}

\subsection{Entrainment and Mixing}

[60] Previous experiments in the area of the entrainment process and dynamics of a gravity current showed that
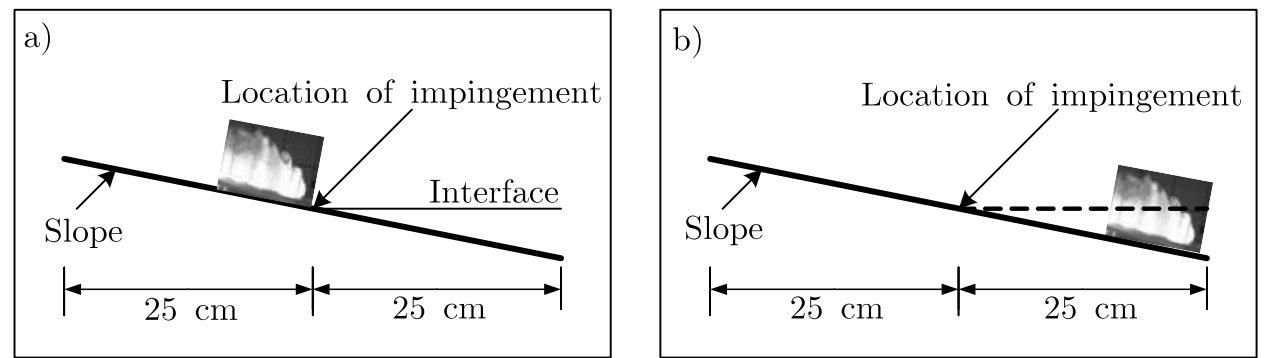

Figure 12. Schematic of the location of the gravity current head for the vorticity fields (Figures 13 and 14) and velocity fields (Figure 18). 

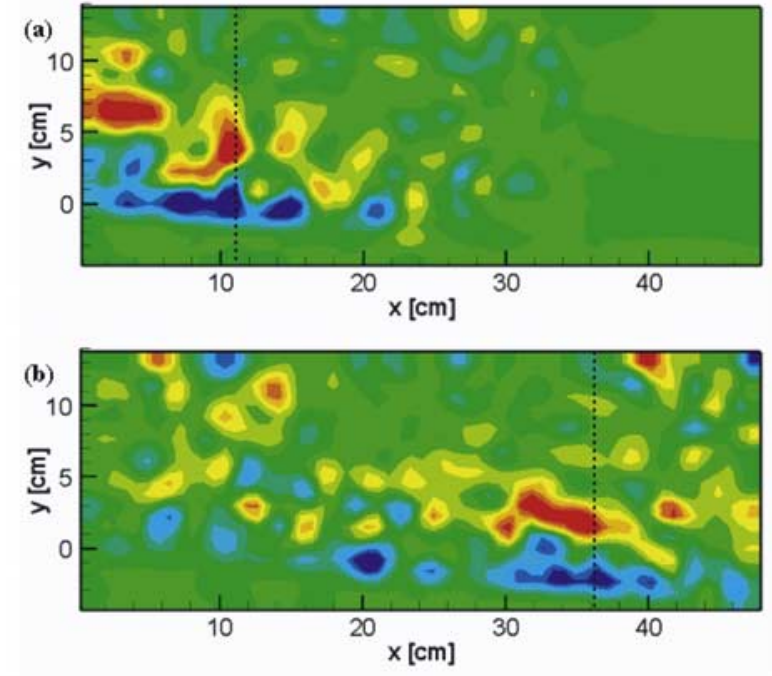

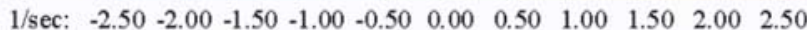

Figure 13. Vorticity field, before (a) and after (b) the impingement for $R i_{(B-T)}=0.5$.

entrainment depends on the Richardson number and on the slope angle [Ellison and Turner, 1959; Britter and Linden, 1980]. Both studies were performed with sloping constant flux currents, in a uniform density environment. Moreover in the case of turbulent flows impinging on a stratified interface, the relationship between turbulent entrainment and Richardson number obeys a power law [Turner, 1973] and depends on Reynolds, Richardson, Schmidt numbers and the Persistence parameter [Cotel and Breidenthal, 1997].

[61] In the case of a gravity current impinging on a stratified interface, we found that the entrainment and mixing are dominated by the action of the KelvinHelmholtz vortices on the back of the head of the gravity current and the entrainment is found to be proportional to $R i_{(B-C)}^{-1}$ which agrees with Cotel and Breidenthal [1997] model for a shear dominated regime. $R i_{(B-C)}$, is defined by the density difference between the current and bottom layer fluid. The mixing rate is also proportional to $R i_{(B-C)}^{-1}$ since the Reynolds number range is above the mixing transition which occurs at Re of around 3,000. The mixing increases by an order of magnitude at this value of Reynolds number and it is the onset of three dimensional instabilities [Breidenthal, 1981]. It has been postulated before [Breidenthal and Baker, 1985; Cotel et al., 1997] that if the Reynolds number is above the mixing transition, then the entrainment and mixing are proportional to each other.

[62] This entrainment power law has been observed for shear driven entrainment by Narimousa and Fernando [1987] and by Price et al. [1978]. The first deals with the entrainment of a two-layer fluid subjected to interfacial velocity shear in laboratory experiments. The latter presents observations from two cases of storm-driven mixing layer deepening in the ocean. All field and laboratory experiments, including the present study, where shear is the dominant mixing mechanism, have reported a $R i^{-1}$ relationship. As explained by Cotel and Breidenthal [1997], when the stratified interface becomes thick under the act of shear, the eddies with an eddy $R i$ equal to unity become the most efficient at entrainment. Dimensional analysis based on this physical argument leads to a $R i^{-1}$ power law.

\subsection{Structure of the Gravity Current Head: Velocity and Vorticity}

[63] From the qualitative observations of the shadowgraph images in Figures 9 and 10, it is obvious that the presence of the stratified interface has a significant effect on the structure of the gravity current head. We observed that the presence of the stratified interface is causing a thickening of the head. In addition the distinctive raised head structure is not observed. More is revealed by a careful analysis of the velocity and vorticity data.

[64] For the weakly stratified case (low Richardson number), the gravity current easily penetrates past the interface, as the density difference between the fluids of the two layers is small and the velocity of gravity current is high. The density difference between the head fluid and the ambient fluid is high enough to create baroclinic vorticity. In addition to that, shear stresses are present between the upper surface of the gravity current and the ambient fluid. These are particularly strong in the case of low $R i$, since the velocity of the gravity current is the highest. As both types of vorticity are rotating counter clockwise, it is therefore difficult with our present measurement techniques to distinguish between the two. These counterclockwise vortices in the upper surface of the head are seen in the vorticity field of Figure 13b. For low Richardson numbers (Figures 16a and 16b) the strength of the vortices is increasing downstream of the impingement zone. The quantities $\Delta \omega^{+}$and $\Delta \omega^{-}$that represent the change in vorticity after and before the impingement, are positive and increasing as the stratification across the interface is decreasing. The change in velocity, $\Delta u$, follows a similar pattern (i.e. it is positive and increasing as the density difference across the interface is
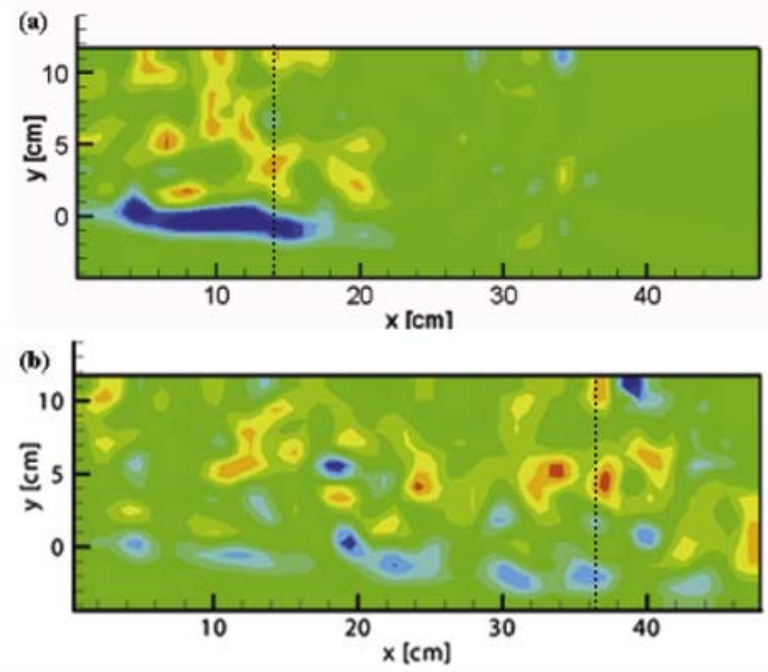

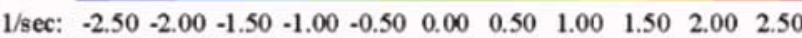

Figure 14. Vorticity field, before (a) and after (b) the impingement for $R i_{(B-T)}=3.0$. 


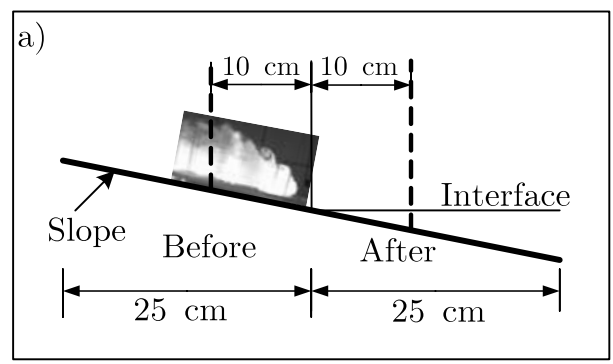

Figure 15. Locations of the vertical profiles of vorticity and velocity (Figures 16 and 19).

decreasing). The velocity of the gravity current is high before the impingement and the density difference across the interface is small. The gravity current easily penetrates the interface and its velocity increases within the range of equation (3).

[65] On the other hand, for the higher Richardson number case, the interface has a significant impact on the current. The velocity of the head (as seen in Figures $19 b$ and 19d) is decreasing. The quantity $\Delta u$ is negative, corresponding to a reduction in velocity. This reduction is becoming larger as the stratification across the interface is increasing (i.e. increasing $R i_{(B-T)}$ number). The vorticity is also significantly decreased beyond the impingement region. In this case, $\Delta \omega^{+}$and $\Delta \omega^{-}$, are negative and the reduction in vorticity is larger as the the stratification across the interface is increasing. This corroborates the entrainment measurements (Figures 5 and 6) where the entrainment rate decreased as the Richardson number increased. In addition,

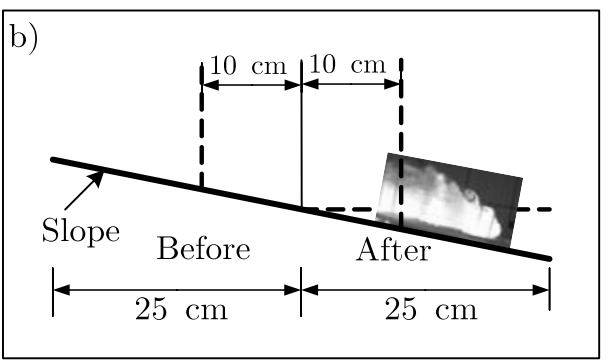

increase in the thickness of the gravity current head was observed in shadowgraph images of the experiments. This thickening occurred after impingement for high $R i$. This evidence supports the observed slow-down of the gravity current (in the presence of a strong stratified interface), as the discharge is kept constant during an experiment.

[66] In summary, as the Ri number increases the gravity current slows down, its vorticity decreases, therefore reducing the rate of entrainment of lower fluid into the gravity current. The Kelvin-Helmholtz vortices are responsible for the entrainment and mixing, therefore if their strength decreases, the entrainment will also have to decrease.

\section{Conclusions}

[67] Gravity currents are an important area of turbulent stratified flows. There has been extensive laboratory, field, analytical/numerical work performed in this area [Simpson,
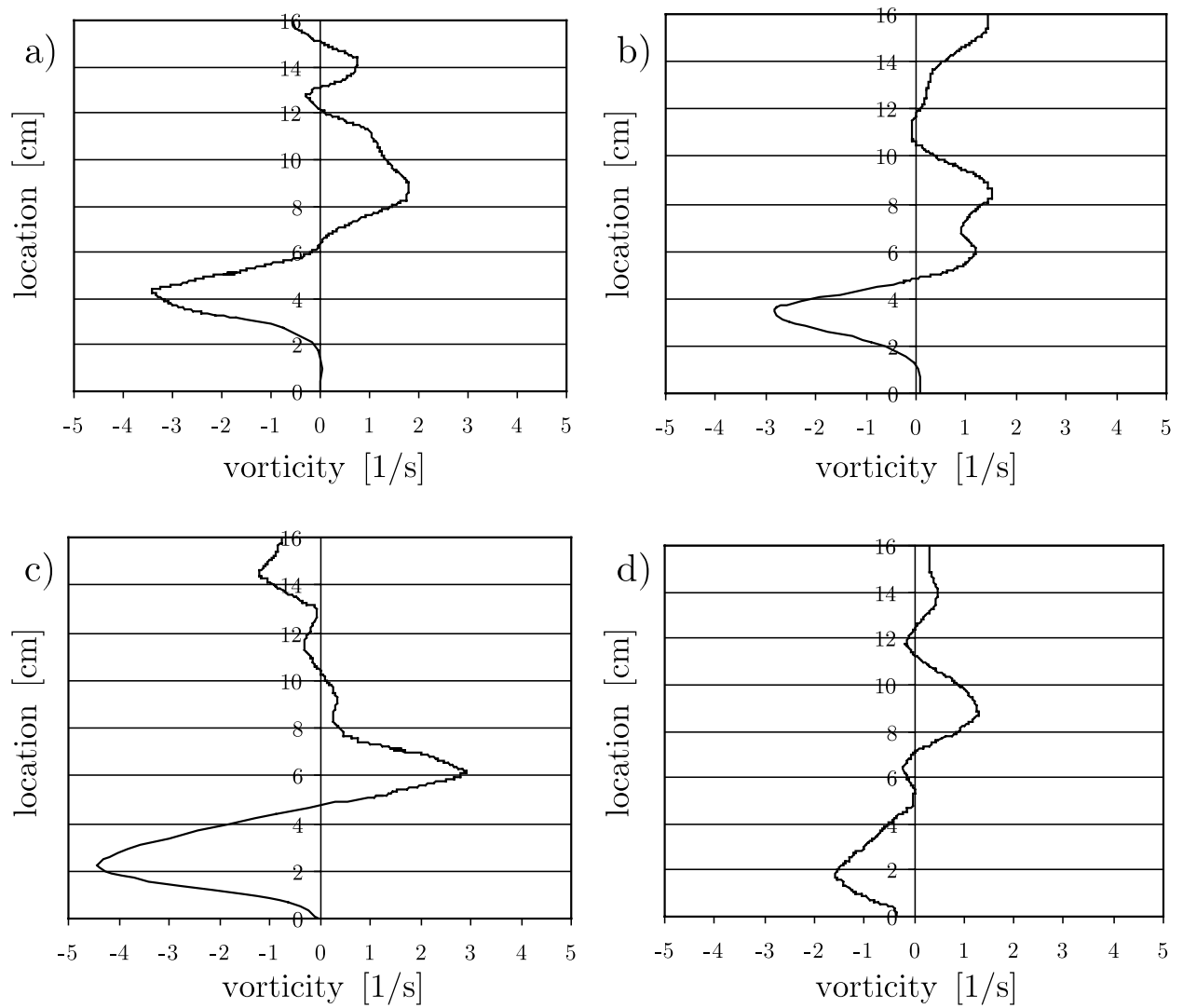

Figure 16. Vertical profiles of vorticity, before $(\mathrm{a}, \mathrm{b})$ and after $(\mathrm{c}, \mathrm{d})$ the impingement for $\operatorname{Ri}_{(B-T)}=0.5$ $(\mathrm{a}, \mathrm{c})$ and $R i_{(B-T)}=3.0(\mathrm{~b}, \mathrm{~d})$. 


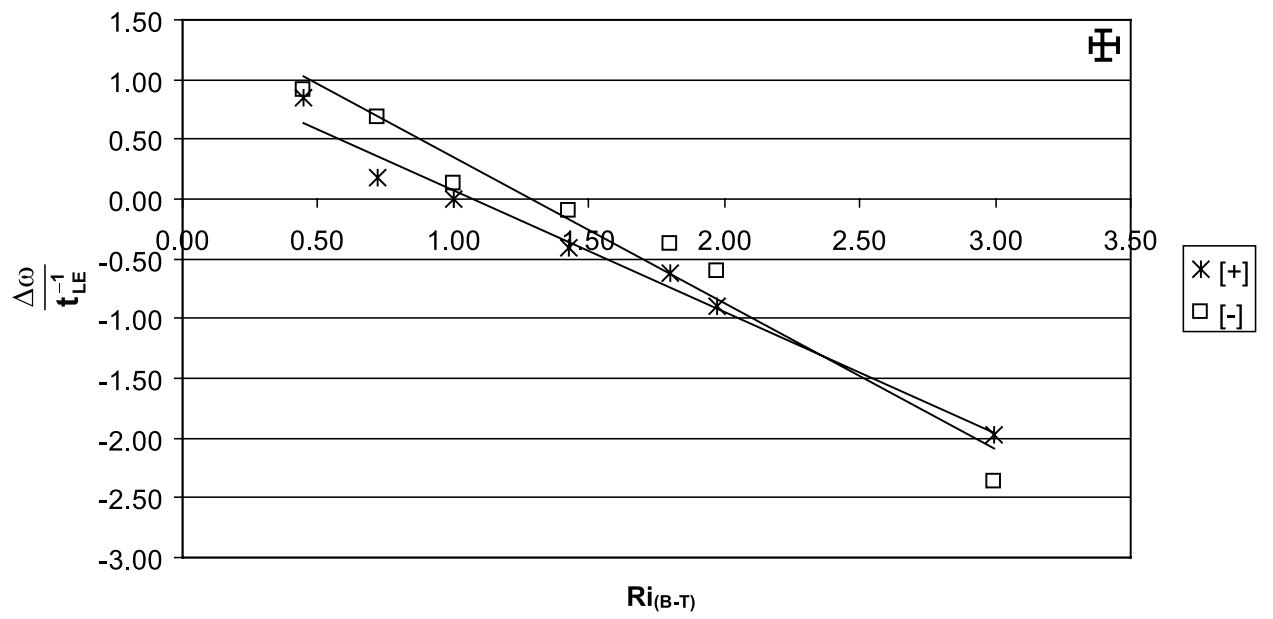

Figure 17. The change of vorticity, non-dimensionalized by the time scale of the large eddies, as a function of $R i_{(B-T)}$.

1997]. The experiments performed in this paper are the first to combine a constant flux current propagating on a slope and entering a two-layer stratified environment. We specifically investigated the effect of a stratified interface on the entrainment/mixing processes and the vortical structure of the gravity current.

[68] For the case of a two layer stratified environment and for a slope angle $\vartheta=6^{\circ}$, we found that:

$$
E \propto R i_{(B-C)}^{-1}
$$

This dependence of entrainment rate on Richardson number has been observed for shear driven entrainment by Narimousa and Fernando [1987] and by Price et al. [1978]. In the case of the example given in the introduction (katabatic wind impinging on an atmospheric inversion over a city), the entrainment/mixing results can be used to predict concentrations of pollutants after the impingement.

[69] Parker et al. [1987] found a similar relationship for turbidity currents moving down a slope of $3^{\circ}$ to $5^{\circ}$ over an erodible bed:

$$
E \propto R i^{-1.2}
$$

In the case of the cascading of cold water in lakes [Fer et $a l ., 2002]$ or the turbitidy currents in the ocean, the entrainment results can be used to predict the concentration of sediments in the current. Furthermore the entrainment and mixing are dominated by the action of the KelvinHelmholtz vortices on the back of the head of the gravity current.

[70] Shadowgraph experiments were performed on the head of the gravity current and the same characteristic raised head, followed by a shallower steady current was observed in Figures 9a and 10a (no stratification), as seen by Britter and Linden [1980]. But in Figure 9b (stratified case) the characteristic raised head is not observed, a clear indication of the effect of the interface on the structure of the head. The interaction of the gravity current and stratified interface results in a slowing-down of the current front as well as an increased head size.
[71] Detailed measurements of the velocity and vorticity fields at the head of the gravity current are provided and the effect of the stratified interface is investigated. For the weakly stratified case, strong counterclockwise vortices in the upper region of the head are generated after impingement. In addition, the velocity increases after the impingement. The quantities $\Delta \omega^{+}, \Delta \omega^{-}$and $\Delta u$ that represent the change in positive, negative vorticity and velocity respectively after and before the impingement, are positive and increasing as the stratification across the interface is decreasing. For the higher Richardson number case both the velocity and the vorticity of the head are significantly decreased beyond the impingement region, indicating a strong effect of the stratified interface on the current dynamics. The quantities $\Delta \omega^{+}, \Delta \omega^{-}$and $\Delta u$ are negative and the reduction in vorticity and velocity is larger as the the
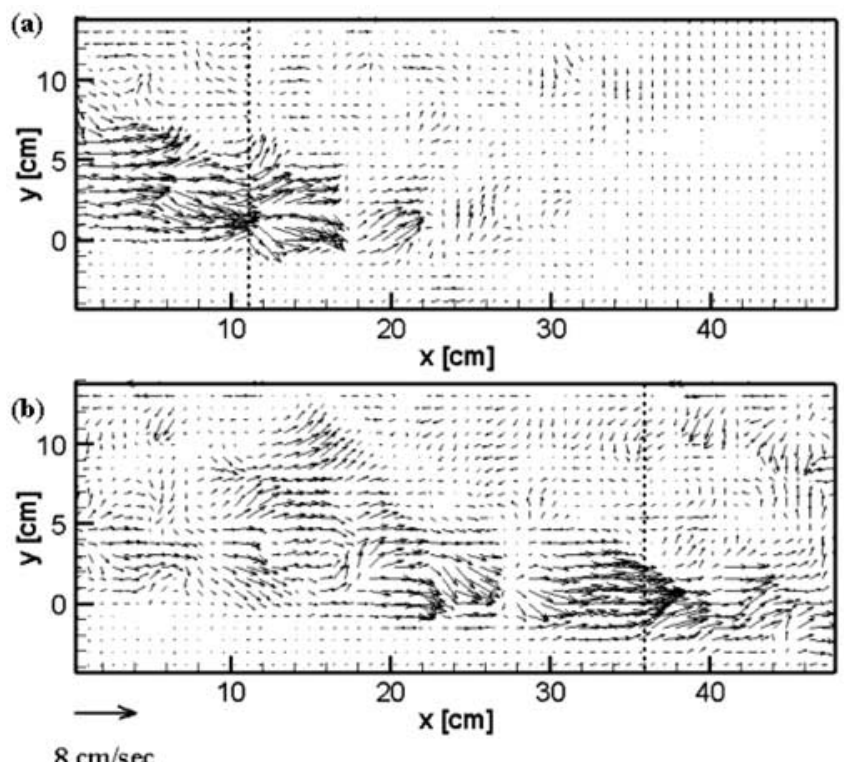

$8 \mathrm{~cm} / \mathrm{sec}$

Figure 18. Velocity field, before (a) and after (b) the impingement for $R i_{(B-T)}=0.5$. 



Figure 19. Vertical profiles of velocity, before (a, b) and after (c, d) the impingement for $\operatorname{Ri}_{(B-T)}=0.5$ $(\mathrm{a}, \mathrm{c})$ and $R i_{(B-T)}=3.0(\mathrm{~b}, \mathrm{~d})$.

stratification across the interface is increasing. The dependence of $\Delta \omega^{+}, \Delta \omega^{-}, \Delta u$ on the Richardson number, $R i_{(B-T)}$, is found to be linear. Again for the example of the katabatic wind, the velocity results can be used to predict the easiness by which the wind is penetrating the inversion or the change in the velocity can be estimated as a result of the impingement.

[72] Before applying these results on natural gravity currents, it should be investigated whether rotation can be ignored based on the time scale of the flow under consideration. For our experimental case the Rossby number is in the order of $10^{5}$ and the effect of rotation is ignored. For rotation to be important the time scale of the flow has to be proportional to $f^{-1}$, where $f=2 \Omega \sin$ (latitude) and $\Omega$ is the angular rate of the earth; so the time scale of the flow would have to be around $7000 \mathrm{sec}$ (around $2 \mathrm{hr}$ ). In our case the gravity current reaches the interface in 12 to $20 \mathrm{sec}$ (for high and low velocity currents respectively) and then the bottom of the tank in other 12 to $20 \mathrm{sec}$. The propagation of the current along the sloped surface is around 25 to $40 \mathrm{sec}$, much less than the time scale of $7000 \mathrm{sec}$ for rotation to be important. From the velocity and length scales of a natural system, a time scale can be evaluated and compared to the limit of $7000 \mathrm{sec}$. If we look at the winter cascading of cold water at lake Geneva [Fer et al., 2002], the mean velocity of the gravity current is $5.2 \mathrm{~cm} / \mathrm{sec}$. When considering the flow in the near shore region (length of $250 \mathrm{~m}$ and slope of $1^{\circ}$ ), the time scale is around $4800 \mathrm{sec}$ and rotation is not important. If the whole slope of the lake is considered
(1250 $\mathrm{m}$ length and varying slope) the time scale is around $6.5 \mathrm{hr}$ and rotation should be considered.

[73] The applications of this work are varied from global climate change to pollution prediction and control. In the case of the example given in the introduction (katabatic wind impinging on an atmospheric inversion over a city), the entrainment/mixing results can be used to predict concentrations of pollutants after the impingement and the velocity/vorticity measurements will provide energy and momentum information necessary to predict penetration of such flows. In the case of turbitidy currents in the ocean or

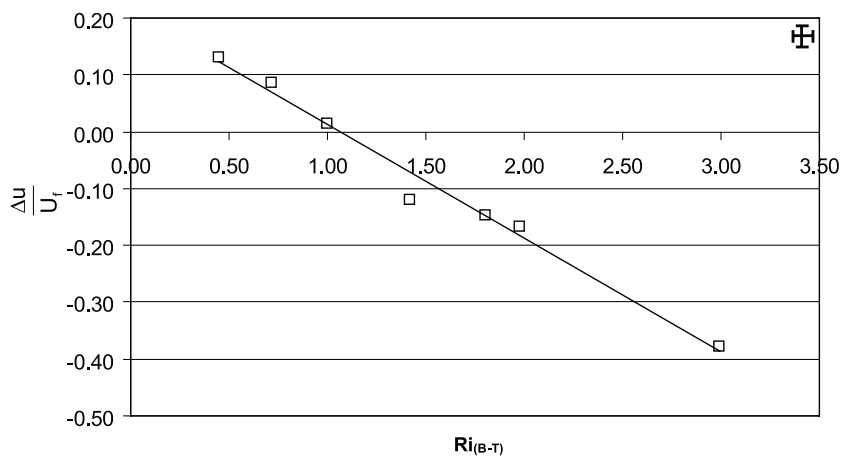

Figure 20. The change of velocity before and after the impingement, non-dimensionalized by the velocity of the head $U_{f}$, as a function of $R i_{(B-T)}$. 
a)

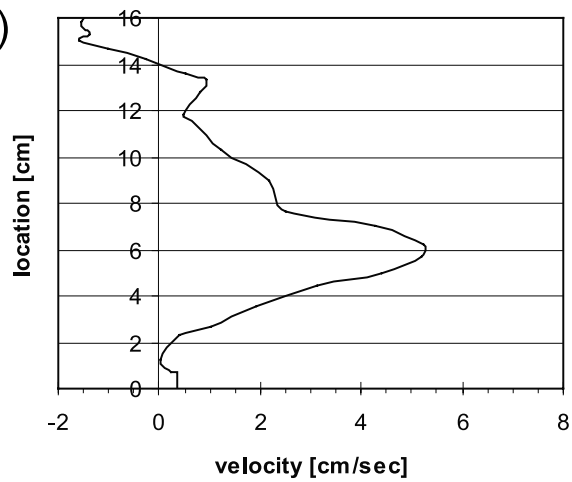

c)

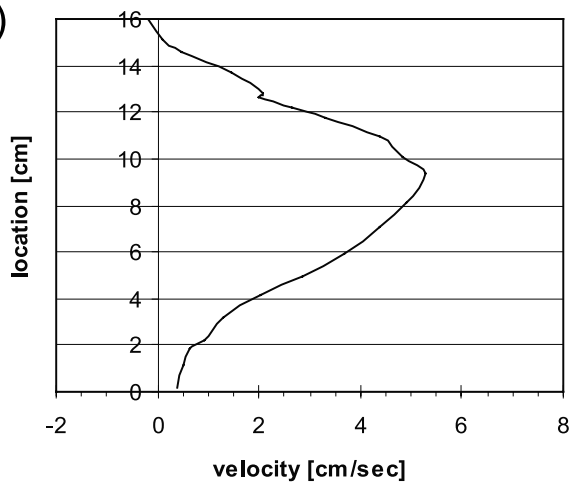

b)

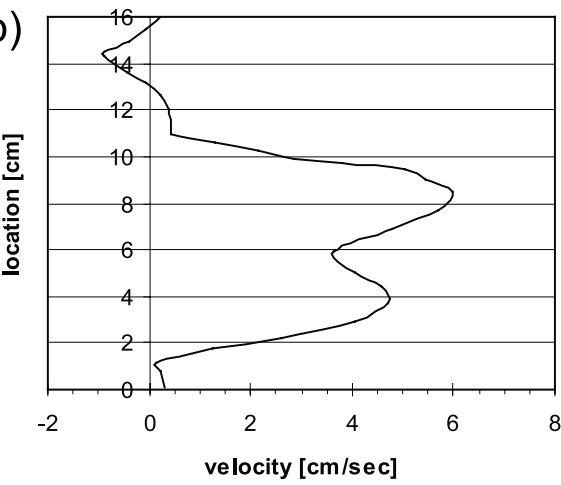

d)



Figure 21. Vertical profiles of velocity for the unstratified case. Figures $21 \mathrm{a}$ and $21 \mathrm{~b}$ correspond to the before impingement location, and Figures $21 \mathrm{c}$ and $21 \mathrm{~d}$ correspond to the after. The density of the gravity current fluid is (a, c) $\rho_{c}=1012 \mathrm{~kg} / \mathrm{m}^{3}$ and $(b, d) \rho_{c}=1022 \mathrm{~kg} / \mathrm{m}^{3}$.

the cascading of cold water in lakes, our entrainment results can be used to predict, to a first approximation, the concentration of sediments in the current and provide a better understanding of the dynamics of these currents.

\section{References}

Adrian, R. J. (1986), Multi-point optical measurements of simultaneous vectors in an unsteady flow - A review, Int. J. Heat Fluid Flow, 7, $127-145$.

Adrian, R. J. (1991), Particle-imaging techniques for experimental fluid mechanics, Annu. Rev. Fluid Mech., 22, 261-304.

Alahyari, A. A., and E. K. Longmire (1996), Development and structure of a gravity current head, Exp. Fluids, 20, 410-416.

Baines, P. G. (2001), Mixing in flows down genlte slopes into stratified environment, J. Fluid Mech., 443, 237-270.

Beghin, P., E. J. Hopfinger, and R. E. Britter (1981), Gravitational convection from instantaneous sources on inclined boundaries, J. Fluid Mech., $107,407-422$.

Benjamin, T. B. (1968), Gravity currents and related phenomena, J. Fluid Mech., 31, 209-248.

Bonnecaze, R. T., and J. R. Lister (1999), Particle-driven gravity currents down planar slopes, J. Fluid Mech., 390, 75-91.

Bonnecaze, R. T., M. A. Hallworth, H. E. Huppert, and J. R. Lister (1995), Axisymmetric particle-driven gravity currents, J. Fluid Mech., 294, $93-$ 121

Breidenthal, R. (1981), Structure in turbulent mixing layers and wakes using a chemical reaction, J. Fluid Mech., 109, 1-24.

Breidenthal, R. E., and M. B. Baker (1985), Convection and entrainment across stratified interfaces, J. Geophys. Res., 90(D7), 13,055-13,062.

Britter, R. E., and P. F. Linden (1980), The motion of the front of a gravity current traveling down an incline, J. Fluid Mech., 99, 531-543.

Britter, R. E., and J. E. Simpson (1978), Experiments on the dynamics of a gravity current head, J. Fluid Mech., 88, 223-240.

Brown, G. L., and A. Roshko (1974), On density effects and large structure turbulence, J. Fluid Mech., 64, 775-816.
Cotel, A. J., and R. E. Breidenthal (1997), A model of vortex persistence effects in stratified entrainment, Appl. Sci. Res., 57, 349-366.

Cotel, A. J., J. A. Gjestvang, N. N. Ramkhelawan, and R. E. Breidenthal (1997), Laboratory experiments of a jet impinging on a stratified interface, Exp. Fluids, 23(2), 155-160.

Dahm, W. J. A., and P. E. Dimotakis (1987), Measurements of entrainment and mixing in turbulent jets, AIAA J., 25, 1216-1223.

Ellison, T. H., and J. S. Turner (1959), Turbulent entrainment in stratified flows, J. Fluid Mech., 4, 432-448.

Fer, I., U. Lemmin, and S. A. Thorpe (2002), Winter cascading of cold water in Lake Geneva, J. Geophys. Res., 107(C6), 3060, doi:10.1029/ 2001JC000828.

Flynn, M. R., and B. R. Sutherland (2004), Intrusive gravity currents and internal gravity wave generation in stratified fluid, J. Fluid Mech., 514, $355-383$.

Hallworth, M. A., H. E. Huppert, J. C. Phillips, and S. J. Sparks (1993), Entrainment in turbulent gravity currents, Nature, 362, 829-831.

Hallworth, M. A., H. E. Huppert, J. C. Phillips, and S. J. Sparks (1996), Entrainment into two dimensional and axisymmetric turbulent gravity currents, J. Fluid Mech., 308, 289-311.

Hallworth, M. A., A. J. Hogg, and H. E. Huppert (1998), Effects of external flow on compostitional and particle gravity currents, J. Fluid Mech., 359, $109-142$.

Hinsch, K. D. (1993), Particle image velocimetry, in Speckle Metrology, edited by R. S. Sirohi, pp. 235-323, Marcel Dekker, New York.

Hopfinger, E. J., and J. C. Tochon-Danguy (1977), A model study of powder-snow avalanches, J. Glasiol., 19, 343-356.

Johari, H. (1992), Mixing in thermals with and without buoyancy reversal, J. Atmos. Sci., 49(16), 1412-1426.

Koochesfahani, M. M., and P. E. Dimotakis (1986), Mixing and chemical reactions in a turbulent mixing layer, J. Fluid Mech., 170, 83112.

McDougall, T. J. (1979), Measurements of turbulence in a zero-mean-shear mixed layer, J. Fluid Mech., 94, 409-431.

Monaghan, J. J., R. A. F. Cas, A. M. Kos, and M. Hallworth (1999), Gravity currents descending a ramp in a stratified tank, J. Fluid Mech., $379,39-69$. 
Narimousa, S., and H. J. S. Fernando (1987), On the sheared density interface of an entraining stratified fluid, J. Fluid Mech., 174, 1-22.

Papantoniou, D., and E. J. List (1989), Large scale structure in the far field of buoyant jets, J. Fluid Mech., 209, 151-190.

Parker, G., M. Garcia, Y. Fukushima, and W. Yu (1987), Experiments on turbidity currents over an erodible bed, J. Hydraul. Res., 25, 123-147.

Price, J. F., C. N. K. Mooers, and J. C. V. Leer (1978), Observation and simulation of storm induced mixed-layer deepening, J. Phys. Oceanogr., $8,582-599$.

Roshko, A. (1976), Structure of turbulent shear flows: A new look, AIAA J., $14,1349-1357$

Ross, A. N., P. F. Linden, and S. B. Dalziel (2002), A study of three dimensional gravity currents on a uniform slope, J. Fluid Mech., 453, $239-261$

Samothrakis, P. (2005), An experimental investigation of the dynamics of a sloping gravity current impinging on a stratified interface, Ph.D. thesis, Univ. of Mich., Ann Arbor.

Simpson, J. E. (1982), Gravity currents in the laboratory, atmosphere, and ocean, Annu. Rev. Fluid Mech., 14, 213-234.
Simpson, J. E. (1997), Gravity Currents in the Environment and the Laboratory, 2nd ed., Cambridge Univ. Press, New York.

Sutherland, B. R., P. J. Kyba, and M. R. Flynn (2004), Intrusive gravity currents in two-layer fluids, J. Fluid Mech., 514, 327-353.

Thomas, L. P., S. B. Dalziel, and B. M. Marino (2003), The structure of the head of an inertial gravity current determined by particle-tracking velocimetry, Exp. Fluids, 34, 708-716.

Thompson, B. W. (1984), Small-scale katabatics and cold hollows, Weather, $41,146-153$

Turner, J. S. (1973), Buoyancy Effects in Fluids, Cambridge Univ. Press, New York.

Valentine, G. A. (1987), Stratified flow in pyroclastic surges, Bull. Volcanol., 49, 616-630.

A. J. Cotel and P. Samothrakis, Department of Civil and Environmental Engineering, University of Michigan, EWRE 19, 1351 Beal Ave., Ann Arbor, MI 48109, USA. (psamothr@umich.edu) 